\title{
Limits of Baumslag-Solitar groups and dimension estimates in the space of marked groups
}

\author{
Luc Guyot and Yves Stalder
}

\begin{abstract}
We prove that the limits of Baumslag-Solitar groups studied by the authors are nonlinear hopfian $\mathrm{C}^{*}$-simple groups with infinitely many twisted conjugacy classes. We exhibit infinite presentations for these groups, classify them up to group isomorphism, describe their automorphisms and discuss the word and conjugacy problems. Finally, we prove that the set of these groups has non-zero Hausdorff dimension in the space of marked groups on two generators.
\end{abstract}

Mathematics Subject Classification (2010). 20E18, 20E06, $20 \mathrm{~F} 10$.

Keywords. Baumslag-Solitar groups, space of marked groups, Turing degree, Hausdorff dimension.

\section{Contents}

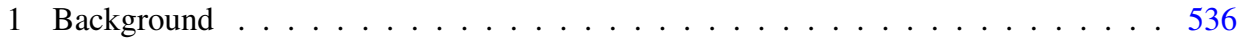

2 HNN decomposition of the limits . . . . . . . . . . . . . . . . . . 539

3 An infinite presentation built up from $\xi \ldots \ldots \ldots \ldots$. . . . . . . . 547

$4 \mathrm{C}^{*}$-simplicity . . . . . . . . . . . . . . . . . . . . . 549

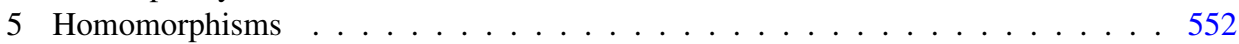

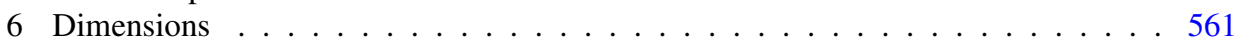

7 Complexity of the word and conjugacy problems . . . . . . . . . 565

References . . . . . . . . . . . . . . . . . . . . 572

\section{Introduction}

The Baumslag-Solitar groups

$$
\mathrm{BS}(p, q)=\left\langle a, b \mid a b^{p} a^{-1}=b^{q}\right\rangle \quad \text { with } p, q \in \mathbb{Z} \backslash\{0\}
$$

are defined in [BS62] and give rise to the first examples of non-hopfian one-relator groups for $p$ and $q$ coprime (a group is called hopfian if it has no isomorphic proper quotient). The groups $\mathrm{BS}(p, q)$ are ubiquitous in group theory and topology [Mol69], 
[Gil79], [FM98], [Why01], [JS79] and offer a remarkable test bed for group-theoretic properties. Considering their limits, we obtain in the present paper a Cantor set of pairwise non-isomorphic two-generated groups with a number of combinatorial and geometrical non-closed properties. By a closed property, we mean a property that defines a closed subset of the space of marked groups. We also give the first estimates of non-vanishing Hausdorff dimension in the space of marked groups.

Let $m \in \mathbb{Z} \backslash\{0\}$. For every sequence $\left(\xi_{n}\right)$ of integers in $\mathbb{Z}$ such that $\left|\xi_{n}\right|$ tends to infinity and $\xi_{n}$ tends to some $m$-adic integer $\xi$, define

$$
\overline{\mathrm{BS}}(m, \xi)=\lim _{n \rightarrow \infty} \mathrm{BS}\left(m, \xi_{n}\right)
$$

where the limit is taken with respect to the topology of the space marked groups; the above sequence being proved to be convergent in [Sta06a], Theorem 6 . Note that $\overline{\mathrm{BS}}(m, n) \neq \mathrm{BS}(m, n)$ for any $n \in \mathbb{Z} \backslash\{0\} \subset \mathbb{Z}_{m}$, for stationary sequences are prohibited. As a consequence of its definition, $\overline{\mathrm{BS}}(m, \xi)$ enjoys the following closed properties: it is torsion-free, it contains a non-abelian free group generated by $b$ and $b a b^{-1}$ if $|m|>1$, and satisfies the relation " $\left[a b^{m} a^{-1}, b\right]=1$ ", which is actually the shortest relation with respect to $a$ and $b$ by [Sta06a], Proposition 2. We present here results of a different nature, relying on the existence of an HNN decomposition.

The limits $\overline{\mathrm{BS}}(m, \xi)$ are first studied for their own right in [GS08], where it is shown that $\overline{\mathrm{BS}}(m, \xi)$ acts transitively on a tree and maps homomorphically onto the special limit $\overline{\mathrm{BS}}(1,0)=\mathbb{Z} \imath \mathbb{Z}$. These two results are extensively used in this article.

Results. We first prove that $\overline{\mathrm{BS}}(m, \xi)$ is a non-degenerate $\mathrm{HNN}$ extension of a free abelian group of infinite countable rank. More precisely, consider the free abelian groups

$$
\begin{aligned}
E & =\mathbb{Z} e_{0} \oplus \mathbb{Z} e_{1} \oplus \mathbb{Z} e_{2} \oplus \cdots, \\
E_{m, \xi} & =\mathbb{Z} m e_{0} \oplus \mathbb{Z}\left(e_{1}-r_{1}(\xi) e_{0}\right) \oplus \mathbb{Z}\left(e_{2}-r_{2}(\xi) e_{0}\right) \oplus \cdots, \\
E_{1} & =\mathbb{Z} e_{1} \oplus \mathbb{Z} e_{2} \oplus \cdots,
\end{aligned}
$$

where $\left(r_{i}(\xi)\right)$ is an integer sequence defined through iterated Euclidean divisions by $m$. Note that the Euclidean division of $x \in \mathbb{Z}_{m}$ by $m$ is well defined since one can write $x=m s+r$ for a unique quotient $s \in \mathbb{Z}_{m}$ and a unique remainder $r \in\{0, \ldots,|m|-1\}$. Set $s_{0}(\xi)=1, r_{0}(\xi)=0$ and apply then Euclidean division of $\xi s_{i}(\xi)$ by $m$ to obtain a new quotient $s_{i+1}(\xi)$ and a new remainder $r_{i+1}(\xi)$. The sequence $\left(r_{i}(\xi)\right)$ is thus defined inductively through the formula $\xi s_{i}(\xi)=m s_{i+1}(\xi)+r_{i+1}(\xi)$. Let $\widetilde{\mathrm{BS}}(m, \xi)$ be the HNN extension of basis $E$ with conjugated subgroups $E_{m, \xi}, E_{1}$ and stable letter $a$, where conjugacy from $E_{m, \xi}$ to $E_{1}$ is defined by $a\left(m e_{0}\right) a^{-1}=e_{1}$ and $a\left(e_{i}-r_{i}(\xi) e_{0}\right) a^{-1}=e_{i+1}$.

Theorem A (Corollary 2.10 and Theorem 3.1). Let $(a, b)$ be the canonical generating pair of $\overline{\mathrm{BS}}(m, \xi)$. Then the map defined by $f(a)=a$ and $f(b)=e_{0}$ induces an 
isomorphism from $\overline{\mathrm{BS}}(m, \xi)$ to $\widetilde{\mathrm{BS}}(m, \xi)$. Moreover, the group $\overline{\mathrm{BS}}(m, \xi)$ admits the infinite presentation

$$
\left\langle a, b \mid\left[b, b_{i}\right]=1, i \geq 1\right\rangle,
$$

with $b_{1}=a b^{m} a^{-1}$ and $b_{i}=a b_{i-1} b^{-r_{i-1}(\xi)} a^{-1}$ for every $i \geq 2$.

In particular, we have $\overline{\mathrm{BS}}(m, 0)=\left\langle a, b \mid\left[a^{i} b^{m} a^{-i}, b\right]=1, i \geq 1\right\rangle$. In addition, the latter presentation is minimal.

Thus Theorem A rules out the only possible exception in [GS08], Theorem 4.1, namely $\xi \in m \mathbb{Z}_{m}$, so that no group $\overline{\mathrm{BS}}(m, \xi)$ can be finitely presented. Note also that $\overline{\mathrm{BS}}(m, \xi)$ enjoys any property shared by all non-degenerate HNN extensions, for instance it is primitive [GG08] and has uniform exponential growth [HB00]. Using the latter HNN decomposition, we show:

Theorem B. Assume that $|m|>1$. Then we have:

- $\overline{\mathrm{BS}}(m, \xi)$ is hopfian but not co-hopfian (Theorem 5.9). If $m$ is prime and $\xi$ is algebraic over $\mathbb{Q}$, then $\overline{\mathrm{BS}}(m, \xi)$ is not residually finite (Proposition 5.10).

- $\overline{\mathrm{BS}}(m, \xi)$ is $C^{*}$-simple and inner-amenable (Proposition 4.1 and Proposition 4.6).

- $\overline{\mathrm{BS}}(m, \xi)$ has infinitely many twisted conjugacy classes (Proposition 5.5).

- $\overline{\mathrm{BS}}(m, \xi)$ is not equationally noetherian and hence not linear (Proposition 5.17).

- The automorphism group of $\overline{\mathrm{BS}}(m, \xi)$ is a split extension of $\overline{\mathrm{BS}}(m, \xi)$ by an infinite dihedral group (Proposition 5.13).

From our study of homomorphisms carried out in Section 5, we deduce the following classification result.

Theorem $\mathbf{C}$ (Theorem 5.11 and Proposition 2.6). Let $m, m^{\prime} \in \mathbb{Z} \backslash\{0\}$ and let $\xi \in \mathbb{Z}_{m}$, $\xi^{\prime} \in \mathbb{Z}_{m^{\prime}}$. Then $\overline{\mathrm{BS}}(m, \xi)$ is abstractly isomorphic to $\overline{\mathrm{BS}}\left(m^{\prime}, \xi^{\prime}\right)$ if and only if there is $\epsilon \in\{ \pm 1\}$ and $d \in \mathbb{N}$ such that $m=\epsilon m^{\prime}, d=\operatorname{gcd}(m, \xi)=\operatorname{gcd}\left(m^{\prime}, \xi^{\prime}\right)$ and the $m$-adic numbers $\xi / d, \epsilon \xi^{\prime} / d$ project onto the same element of $\mathbb{Z}_{m / d}$ via the canonical map $\mathbb{Z}_{m} \rightarrow \mathbb{Z}_{m / d}$.

Thus two given limits are isomorphic if and only if they are isomorphic as marked groups [GS08], Theorem 2.1, i.e., if and only if they represent the same point in the space of marked groups on two generators.

The first-named author has shown that the box-counting dimension of $\mathscr{E}_{2}$, the space of marked groups on two generators, is infinite [Guy07]. Estimating the Hausdorff dimension of $Z_{m}^{\times}$, the set of marked groups $\overline{\mathrm{BS}}(m, \xi)$ such that $\xi$ is invertible in $\mathbb{Z}_{m}$ (Theorem 6.5), we deduce the following:

Theorem D (Corollary 6.6). The Hausdorff dimension of $\mathscr{E}_{2}$ satisfies $\operatorname{dim}_{H}\left(\mathscr{G}_{2}\right) \geqslant$ $\log (2) / 6$. In particular, the Hausdorff dimension of $\mathscr{E}_{2}$ does not vanish. 
Our last results pertain to the algorithmic complexity of the word and conjugacy problem. For every problem that can be suitably represented by a language and every $m$-adic number there is a well-defined degree of complexity, called the Turing degree (Section 7).

Theorem $\mathbf{E}$ (Corollary 7.7). Assume that $\xi$ is invertible in $\mathbb{Z}_{m}$. Then the following Turing degrees coincide:

- the Turing degree of the word problem for $\overline{\mathrm{BS}}(m, \xi)$;

- the Turing degree of the conjugacy problem for $\overline{\mathrm{BS}}(m, \xi)$;

- the Turing degree of $\xi$.

In particular, the word problem is solvable for $\overline{\mathrm{BS}}(m, \xi)$ if and only if $\xi$ is a computable number.

The resolution degree $r_{\Gamma}(n)$ of the word problem for a finitely generated group $\Gamma$ [Gri85], Definition 1, is a quantitative measure of the undecidability of the word problem based on Kolgomorovś ideas. It is, intuitively, the minimal amount of information necessary to decide if $w=1$ in G for every word $w$ with $|w| \leq n$, where $|w|$ is the length of $w$ with respect to a chosen generating set. For a word $w$, we denote by $\operatorname{KR}(w)$ the Kolmogorov complexity resolution of $w$ which is, intuitively, the minimal amount of information necessary to obtain, for every natural number $i \leq|w|$, the $i$-th symbol of the word $w$.

Theorem $\mathbf{F}$ (Proposition 2.5 and Theorem 6.1). Let $\Gamma=\mathrm{BS}(m, \xi)$ and let $\omega=$ $\left(r_{i}(\xi)\right)$. Then $r_{\Gamma}(n)$ is equivalent to $\operatorname{KR}\left(\omega^{(n)}\right)$.

We denote by $\omega^{(n)}$ be the word made of the first $n$ symbols of $\omega$ and say that two functions $f$ and $g$ are equivalent if there is some $C>0$ such that $f(n) \leq g(C n)+C$ and $g(n) \leq f(C n)+C$ for every $n$. For comparison, the resolution degree of a Grigorchuk group $\Gamma=G_{\omega}$ defined in [Gri84] is equivalent to $\operatorname{KR}\left(\omega^{([\log n])}\right)$ [Gri85], Theorem 3, the symbols of $\omega$ assuming there only three possible values. Thus, for a typical $\omega$ in the measure-theoretic sense, $r_{\Gamma}(n)$ is linear for the corresponding $\mathrm{BS}(m, \xi)$ and logarithmic for $G_{\omega}$ [ZL70].

\section{Background}

1.1. The space of marked groups. The commonly used definition of the space of marked groups ${ }^{1}$ is due to Grigorchuk who proved by a topological argument that his intermediate growth groups cannot be finitely presented [Gri84]. The space of marked

\footnotetext{
${ }^{1}$ A very similar topology was first considered in [Gro81], Final remarks. The general idea of topologizing sets of groups goes back to Mahlers and Chabauty [Mah46], [Cha50]. The interested reader should consult [Har08] for a thorough account.
} 
groups has then been used to prove both existence and abundance of groups with exotic properties [Ste96], [Cha00] and has turned to be a remarkably suited framework for the study of Sela's limit groups [CG05]. Isolated points were investigated in [CGP07] and an isolated group is used by de Cornulier to answer a question of Gromov concerning the existence of sofic groups which do not arise as limits of amenable groups [Cor09b]. Very little is known on its topological type; nevertheless the topological type of the space of metabelian marked groups is unveiled in [Cor09a]. The box-counting dimension of the space of marked groups on $k$ generators is infinite if $k \geq 2$ [Guy07]. We show that its Hausdorff dimension is non-zero in Section 6. These computations are carried out using the class of Hölder equivalent metrics defined below.

Definitions. The free group on $k$ generators will be denoted by $\mathbb{F}_{k}$, or $\mathbb{F}(S)$ with $S=\left(s_{1}, \ldots, s_{k}\right)$, if we want to precise the names of canonical generating elements. A marked group on $k$ generators is a pair $(G, S)$ where $G$ is a group and $S=$ $\left(s_{1}, \ldots, s_{k}\right) \in G^{k}$ is an ordered generating set of $G$, also referred to as a marking of $G$. An isomorphism of marked groups is an isomorphism which respects the markings. A marked group $(G, S)$ is endowed with a canonical epimorphism $\phi: \mathbb{F}_{S} \rightarrow G$, which induces an isomorphism of marked groups between $\mathbb{F}_{S} / \operatorname{ker} \phi$ and $G$. Hence a class of marked groups for the relation of marked isomorphism is represented by a unique quotient of $\mathbb{F}_{S}$. The non-trivial elements of $\operatorname{ker} \phi$ are called relations of $(G, S)$. Given $w \in \mathbb{F}_{k}$ we will often write " $w=1$ in $G$ " to say that the image of $w$ in $G$ is trivial.

Let $w=x_{1}^{\varepsilon_{1}} \ldots x_{n}^{\varepsilon_{n}}$ be a reduced word in $\mathbb{F}_{S}$ (with $x_{i} \in S$ and $\varepsilon_{i} \in\{ \pm 1\}$ ). The integer $n$ is called the length of $w$ and denoted $|w|$. If $(G, S)$ is a marked group on $k$ generators and $g \in G$, the length of $g$ is

$$
\begin{aligned}
|g|_{G} & :=\min \left\{n \mid g=s_{1} \ldots s_{n} \text { with } s_{i} \in S \sqcup S^{-1}\right\} \\
& =\min \left\{|w| \mid w \in \mathbb{F}_{S}, \phi(w)=g\right\} .
\end{aligned}
$$

Let $\mathscr{E}_{k}$ be the class of marked groups on $k$ generators up to marked isomorphism. Let us recall that the topology on $\mathscr{E}_{k}$ comes from the following ultrametric distance: for $\left(G_{1}, S_{1}\right) \neq\left(G_{2}, S_{2}\right) \in \mathscr{E}_{k}$ we set $d\left(\left(G_{1}, S_{1}\right),\left(G_{2}, S_{2}\right)\right):=e^{-\lambda}$ where $\lambda$ is the length of a shortest element of $\mathbb{F}_{k}$ which vanishes in one group and not in the other one. Replacing $e$ by $e^{\alpha}$ with $\alpha>0$ in the definition of $d(\cdot, \cdot)$, we obtain an Hölder equivalent metric $d_{\alpha}(\cdot, \cdot)$ with respect to which Hausdorff dimensions are scaled by a factor $\alpha$.

We will use the following characterization of convergent sequences [Sta06a], Proposition 1, without further notice.

Lemma 1.1. Let $\left(G_{n}\right)$ be a sequence of marked groups in $\mathcal{E}_{k}$. The sequence $\left(G_{n}\right)$ converges if and only if for any $w \in \mathbb{F}_{k}$, we have either $w=1$ in $G_{n}$ for $n$ large enough, or $w \neq 1$ in $G_{n}$ for $n$ large enough. 
The free group $\mathbb{F}_{2}=\mathbb{F}(a, b)$, the Baumslag-Solitar groups $\operatorname{BS}(p, q)$ and their limits $\overline{\mathrm{BS}}(m, \xi)$ are marked by their canonical generating pair $(a, b)$.

1.2. The ring of $\boldsymbol{m}$-adic integers. Let $m \in \mathbb{Z} \backslash\{0\}$. Recall that the ring of $m$-adic integers $\mathbb{Z}_{m}$ is the projective limit in the category of topological rings of the system

$$
\cdots \rightarrow \mathbb{Z} / m^{h} \mathbb{Z} \rightarrow \mathbb{Z} / m^{h-1} \mathbb{Z} \rightarrow \cdots \rightarrow \mathbb{Z} / m^{2} \mathbb{Z} \rightarrow \mathbb{Z} / m \mathbb{Z}
$$

where the arrows are the canonical surjective homomorphisms (Note that $\mathbb{Z}_{-m}=\mathbb{Z}_{m}$ and $\mathbb{Z}_{m}$ is the zero ring if $|m|=1$.). The topological ring $\mathbb{Z}_{m}$ is compact since all rings in the above system are finite. Its topology is compatible with the ultrametric distance given, for $\xi \neq \eta$, by

$$
|\xi-\eta|_{m}=|m|^{-\max \left\{k \in \mathbb{N} \mid \xi-\eta \in m^{k} \mathbb{Z}_{m}\right\}},
$$

and $\mathbb{Z}$ embeds isomorphically and densely in $\mathbb{Z}_{m}$. To avoid ambiguity, we call elements of $\mathbb{Z}$ rational integers. We only need the following easy facts about $m$-adic integers. Detailed proofs can be found in the second-named author's Ph.D. thesis [Sta05], Appendix C.

- There is a topological ring isomorphism from $\mathbb{Z}_{m}$ to $\bigoplus_{p \mid m} \mathbb{Z}_{p}$, where $p$ ranges in the set of the prime divisors of $m$.

- Any non-zero ideal can be uniquely written under the form $d \mathbb{Z}_{m}$ where $d$ is a positive rational integer whose prime divisors divide $m$. Moreover we have $\mathbb{Z} \cap d \mathbb{Z}_{m}=d \mathbb{Z}$. For any $n \in \mathbb{Z}$ and any $\xi \in \mathbb{Z}_{m}$, we can define the greatest common divisor $\operatorname{gcd}(n, \xi)$ of $n$ and $\xi$ as the a unique positive rational integer $d$ such that $n$ and $\xi$ generate $d \mathbb{Z}_{m}$.

- An $m$-adic integer $\xi$ is invertible if and only if it does not belong to any of the ideals $p \mathbb{Z}_{m}$ where $p$ is a prime divisor of $m$ in $\mathbb{Z}$; equivalently $\operatorname{gcd}(m, \xi)=1$.

Note finally that non-zero rational integers are never zero divisors in $\mathbb{Z}_{m}$. Hence, we can consider the ring $\mathbb{Z}^{-1} \mathbb{Z}_{m}$ whose elements are fractions of the form $\frac{a}{b}$ with $a \in \mathbb{Z}_{m}$ and $b \in \mathbb{Z} \backslash\{0\}$ and whose laws are the classical ones for fractions.

1.3. HNN extensions and tree actions. In this section we fix our notations for HNN extensions and collect several facts concerning their standard tree actions. Let $G$ be a group, let $H, K$ be subgroups of $G$ and let $\tau: H \rightarrow K$ be an isomorphism. The $H N N$ extension of base $G$ whose stable letter $t$ conjugates $H$ to $K$ via $\tau$, is the group

$$
\left.\operatorname{HNN}(G, H, K, \tau)=\langle G, t| t h t^{-1}=\tau(h) \text { for every } h \in H\right\rangle .
$$

Let $\Gamma=\operatorname{HNN}(G, H, K, \tau)$. We say that $\Gamma$ is an ascending $\mathrm{HNN}$ extension if either $G=H$ or $G=K$. We say that $\Gamma$ is a degenerate HNN extension if $G=H=K$, i.e., $\Gamma=G \rtimes \mathbb{Z}$ where $\langle t\rangle$ identifies with $\mathbb{Z}$. Note that a given group (e.g. $\mathbb{Z} \imath \mathbb{Z}$ ) may have two different $\mathrm{HNN}$ decompositions, one being degenerate whereas the other is not. 
The Normal Form Theorem. A sequence $g_{0}, t^{\epsilon_{1}}, g_{1}, \ldots, t^{\epsilon_{n}}, g_{n}$, with $n \geq 0$, is said to be a reduced sequence if there is no consecutive subsequence $t, g_{i}, t^{-1}$ with $g_{i} \in H$ or $t^{-1}, g_{i}, t$ with $g_{i} \in K$. Britton's lemma [LS77], p. 181, asserts that the word $g_{0} t^{\epsilon_{1}} \ldots t^{\epsilon_{n}} g_{n}$ has a non-trivial image $\gamma$ in $\Gamma$ if $g_{0}, t^{\epsilon_{1}}, \ldots, t^{\epsilon_{n}}, g_{n}$ is reduced and $n \geq 1$. Such a word is called a reduced form for $\gamma$. Although $\gamma$ may have different reduced forms, the sequence $t^{\epsilon_{1}}, \ldots, t^{\epsilon_{n}}$ is uniquely determined by $\gamma$ and we call its length $n=|\gamma|_{t}$ the $t$-length of $\gamma$. Fixing a set $T_{H}$ of representatives of right cosets of $H$ in $G$ and a set $T_{K}$ of representatives of right cosets of $K$ in $G$ such that $1 \in T_{H} \cap T_{K}$, the Normal Form Theorem [LS77], Theorem IV.2.1, asserts that there is a unique sequence $g_{0}, t^{\epsilon_{1}}, \ldots, t^{\epsilon_{n}}, g_{n}$ representing $\gamma \in \Gamma$ with the following properties:

- $g_{0}$ is an arbitrary element of $G$,

- If $\epsilon_{i}=1$, then $g_{i} \in T_{H}$,

- If $\epsilon_{i}=-1$, then $g_{i} \in T_{K}$,

- there is no consecutive subsequence $t^{\epsilon}, 1, t^{-\epsilon}$.

This sequence is the normal sequence of $\gamma$ with respect to $T_{H}$ and $T_{K}$ and we call the word $g_{0} t^{\epsilon_{1}} \ldots t^{\epsilon_{n}} g_{n}$ the normal form of $\gamma$.

Action on the Bass-Serre tree and its boundary. The Bass-Serre tree of $\Gamma=$ $\operatorname{HNN}(G, H, K, \tau)$ is the oriented tree $T$ with vertex set $V(T)=\Gamma / G$, with set of oriented edges $E_{+}(T)=\Gamma / H$ subject to the incidence relations $o(\gamma H)=\gamma G$ and $t(\gamma H)=\gamma t^{-1} G$.

Given a tree $T$, we define the boundary $\partial T$ as the set of cofinal rays. The set $\partial T$ has a natural topology defined as follows. We call a shadow the boundary of any connected component of $T$ deprived of one of its edges. The family of shadows generates a topology on $\partial T$ which is Hausdorff and totally discontinuous. If $T$ is a countable non-linear tree, e.g. the standard Bass-Serre tree of a non-degenerate HNN extension, then $\partial T$ is a perfect Baire space [HP11], Proposition 10 and 20.ii'. Note that every automorphism of $T$ induces an homeomorphism of $\partial T$.

As $\Gamma$ acts without inversion on its Bass-Serre tree $T$, every element $\gamma \in \Gamma$, viewed as a tree automorphism, is either elliptic, i.e., $\gamma$ fixes some vertex of $T$, or hyperbolic, i.e., $\gamma$ fixes no vertex of $T$ but exactly two ends of $\partial T$ [Ser77]. The action of $\Gamma$ on $\partial T$ has no fixed end if $\Gamma$ is non-ascending, exactly one fixed end if $\Gamma$ is non-degenerate and ascending, and exactly two fixed ends if $\Gamma$ is degenerate [HP11], Proposition 20.

\section{HNN decomposition of the limits}

We fix $m \in \mathbb{Z} \backslash\{0\}, \xi \in \mathbb{Z}_{m}$ and $\left(\xi_{n}\right)$ a sequence of rational integers such that $\left|\xi_{n}\right| \rightarrow \infty$ and $\xi_{n} \rightarrow \xi$ in $\mathbb{Z}_{m}$. A natural HNN decomposition arises from the transitive action of $\overline{\mathrm{BS}}(m, \xi)$ on a tree constructed in [GS08]. We recall this construction. 
We denote by $H_{n}$ (respectively $\left.H_{n}^{m}\right)$ the subgroup of $\operatorname{BS}\left(m, \xi_{n}\right)$ generated by $b$ (respectively $\left.b^{m}\right)$ and by $T_{n}$ the Bass-Serre tree of $\mathrm{BS}\left(m, \xi_{n}\right)$. We set

$$
\begin{gathered}
Y=\left(\prod_{n \in \mathbb{N}} V\left(T_{n}\right)\right) / \sim=\left(\prod_{n \in \mathbb{N}} \mathrm{BS}\left(m, \xi_{n}\right) / H_{n}\right) / \sim, \\
Y^{m}=\left(\prod_{n \in \mathbb{N}} E_{+}\left(T_{n}\right)\right) / \sim=\left(\prod_{n \in \mathbb{N}} \mathrm{BS}\left(m, \xi_{n}\right) / H_{n}^{m}\right) / \sim,
\end{gathered}
$$

where $\sim$ is defined by $\left(x_{n}\right) \sim\left(y_{n}\right) \Longleftrightarrow \exists n_{0} \forall n \geqslant n_{0}: x_{n}=y_{n}$ in both cases. We now define an oriented graph $X=X_{m, \xi}$ by

$V(X)=\left\{x \in Y \mid \exists w \in \mathbb{F}_{2}\right.$ such that $\left.\left(x_{n}\right) \sim\left(w H_{n}\right)\right\}=\left\{\left(w H_{n}\right) \mid w \in \mathbb{F}_{2}\right\} / \sim$, $E_{+}(X)=\left\{y \in Y^{m} \mid \exists w \in \mathbb{F}_{2}\right.$ such that $\left.\left(y_{n}\right) \sim\left(w H_{n}^{m}\right)\right\}=\left\{\left(w H_{n}^{m}\right) \mid w \in \mathbb{F}_{2}\right\} / \sim$, $\left.o\left(w H_{n}^{m}\right)\right)=\left(w H_{n}\right)=\left(o\left(w H_{n}^{m}\right)\right)$, $t\left(\left(w H_{n}^{m}\right)\right)=\left(w a^{-1} H_{n}\right)=\left(t\left(w H_{n}^{m}\right)\right)$.

The graph $X_{m, \xi}$ is a tree and the obvious action of $\mathbb{F}_{2}$ on $X_{m, \xi}$ factorizes through the canonical projection $\mathbb{F}_{2} \rightarrow \overline{\mathrm{BS}}(m, \xi)$ [GS08], Section 3. Let $v_{0}=\left(H_{n}\right)$ and $e_{0}=\left(H_{n}^{m}\right)$. We denote by $B$ (respectively $B_{m}$ ) the stabilizer of $v_{0}$ (respectively $e_{0}$ ) in $\overline{\mathrm{BS}}(m, \xi)$. We set $B_{\xi}=a B_{m} a^{-1}$. As the action of $\overline{\mathrm{BS}}(m, \xi)$ is clearly transitive on vertices and geometrical edges, the group $\overline{\mathrm{BS}}(m, \xi)$ has a HNN decomposition:

Proposition 2.1. The group $\overline{\mathrm{BS}}(m, \xi)$ is the HNN extension of base group $B$, stable letter $a$ and conjugated subgroups $B_{m}$ and $B_{\xi}=a B_{m} a^{-1}$.

We define the inner automorphism $\tau_{a}: \gamma \mapsto a \gamma a^{-1}$ of $\overline{\mathrm{BS}}(m, \xi)$ and we denote by $\operatorname{HNN}\left(B, B_{m}, B_{\xi}, \tau\right)$ the previous HNN decomposition where $\tau$ is the isomorphism from $B_{m}$ to $B_{\xi}$ induced by $\tau_{a}$.

Proof. This follows from results in [Ser77]. See in particular Section I.5 and Remark 1 after Theorem 7 in Section I.4.

The following lemma is an immediate consequence of the definition of the action.

Lemma 2.2. Let $w \in \mathbb{F}_{2}$ and let $\gamma$ be its image in $\overline{\mathrm{BS}}(m, \xi)$. Then $\gamma \in B$ (respectively $\left.\gamma \in B_{m}\right)$ if and only if there is rational integer sequence $\left(\lambda_{n}\right)$ such that $w=b^{\lambda_{n}}$ (respectively $\left.b^{m \lambda_{n}}\right)$ in $\mathrm{BS}\left(m, \xi_{n}\right)$ for all $n$ large enough.

As a result, $B$ is abelian. Let $\mathbb{Z}[X]$ be the ring of polynomials in the variable $X$ with integer coefficients. A thorough study of the sequence $\left(\lambda_{n}\right)$ of Lemma 2.2 enables us to embed $B$ isomorphically into $\mathbb{Z}[X]$ seen as an abelian group:

Proposition 2.3. Let $w \in \mathbb{F}_{2}$ with image $\gamma \in B$. Then there is a unique polynomial $P_{\gamma}(X) \in \mathbb{Z}[X]$ such that $w=b^{P_{\gamma}\left(\xi_{n} / m\right)}$ in $\mathrm{BS}\left(m, \xi_{n}\right)$ for all $n$ large enough.

The map $q: \gamma \mapsto P_{\gamma}(X)$ defines an injective homomorphism from $B$ into $\mathbb{Z}[X]$. 
The construction of the homomorphism $q$ relies on a sequence of polynomials built up from $m$ and $\xi$ in a non obvious way. Its definition is the subject of the next section. The proof of Proposition 2.3 is therefore postponed to Section 2.2 (see Proposition 2.9).

2.1. The functions $\boldsymbol{r}_{\boldsymbol{i}}$ and $s_{\boldsymbol{i}}$. In this section we define functions $r_{i}$ and $s_{i}$ on $\mathbb{Z}$ which describe how the $b$ exponents of a given word $w \in \mathbb{F}_{2}$ behave when we reduce it in $\mathrm{BS}(m, n)$ for $n$ ranging in a given class modulo $m^{h}$. In [Sta06a], [GS08], these functions were decisive to describe converging sequences of Baumslag-Solitar groups. It turns out that they extend continuously to $\mathbb{Z}_{m}$ and that $\xi$, and hence $\overline{\mathrm{BS}}(m, \xi)$, is uniquely determined by $m$ and the sequence $\left(r_{i}(\xi)\right)$.

Definition 2.4. We define the functions $r_{0}, r_{1}, \ldots, s_{0}, s_{1}, \ldots$ on $\mathbb{Z}$ depending on a parameter $m \in \mathbb{Z} \backslash\{0\}$ by the inductive formulas

$$
\begin{gathered}
r_{0}(n)=0, \quad s_{0}(n)=1 ; \\
n s_{i-1}(n)=m s_{i}(n)+r_{i}(n) \text { with } r_{i}(n) \in\{0, \ldots,|m|-1\} \text { for every } i \geq 1 .
\end{gathered}
$$

Proposition 2.5. Let $n, n^{\prime} \in \mathbb{Z}$ such that $\operatorname{gcd}(n, m)=\operatorname{gcd}\left(n^{\prime}, m\right)=d$. Let $h \in$ $\mathbb{N} \backslash\{0\}$ and $\hat{m}=m / d$.

(1) Assume that $n \equiv n^{\prime}\left(\bmod \hat{m}^{h} d \mathbb{Z}\right)$. Then the following holds:

(i) $r_{i}(n)=r_{i}\left(n^{\prime}\right)$ for $i=1, \ldots, h$;

(ii) $s_{i}(n) \equiv s_{i}\left(n^{\prime}\right)\left(\bmod \hat{m}^{h-i} \mathbb{Z}\right)$ for $i=1, \ldots, h$.

(2) If $r_{i}(n)=r_{i}\left(n^{\prime}\right)$ for $i=1, \ldots, h$, then $n \equiv n^{\prime}\left(\bmod \hat{m}^{h} d \mathbb{Z}\right)$.

Proof of Proposition 2.5. Proof of (1). We show by induction on $i$ that $r_{i}(n)=$ $r_{i}\left(n^{\prime}\right), s_{i}(n) \equiv s_{i}\left(n^{\prime}\right)\left(\bmod \hat{m}^{h-i} \mathbb{Z}\right)$ and $s_{i}(n) \cdot n \equiv s_{i}\left(n^{\prime}\right) \cdot n^{\prime}\left(\bmod \hat{m}^{h-i} d \mathbb{Z}\right)$ for $i=0,1, \ldots, h$. The case $i=0$ is obvious. Assume that $r_{i-1}(n)=r_{i-1}\left(n^{\prime}\right)$, $s_{i-1}(n) \equiv s_{i-1}\left(n^{\prime}\right)\left(\bmod \hat{m}^{h-i+1} \mathbb{Z}\right)$ and $s_{i-1}(n) \cdot n \equiv s_{i-1}\left(n^{\prime}\right) \cdot n^{\prime}\left(\bmod \hat{m}^{h-i+1} d \mathbb{Z}\right)$ for some $1 \leq i \leq h$. By construction

$$
s_{i-1}(n) \cdot n=s_{i}(n) \cdot m+r_{i}(n)
$$

and

$$
s_{i-1}\left(n^{\prime}\right) \cdot n^{\prime}=s_{i}\left(n^{\prime}\right) \cdot m+r_{i}\left(n^{\prime}\right) .
$$

We deduce from the induction hypothesis that $r_{i}(n) \equiv r_{i}^{\prime}\left(n^{\prime}\right)(\bmod m \mathbb{Z})$ and hence $r_{i}(n)=r_{i}\left(n^{\prime}\right)$. As a result

$$
s_{i}(n)-s_{i}\left(n^{\prime}\right)=\frac{\left(s_{i-1}(n) \cdot n-s_{i-1}\left(n^{\prime}\right) \cdot n^{\prime}\right)}{m},
$$

from which we deduce that

$$
s_{i}(n) \equiv s_{i}\left(n^{\prime}\right) \quad\left(\bmod \hat{m}^{h-i} \mathbb{Z}\right)
$$


still by using the induction hypothesis. Look at the right member of the relation

$$
s_{i}(n) \cdot n-s_{i}\left(n^{\prime}\right) \cdot n^{\prime}=\left(s_{i}(n)-s_{i}\left(n^{\prime}\right)\right) \cdot n+s_{i}\left(n^{\prime}\right) \cdot\left(n-n^{\prime}\right) .
$$

The first term is a multiple of $\hat{m}^{h-i} d$ because of equation (1) and the fact that $d$ divides $n$. The second one is a multiple of $\hat{m}^{h-i} d$ because of hypothesis (1). Therefore, we get $s_{i}(n) \cdot n \equiv s_{i}\left(n^{\prime}\right) \cdot n^{\prime}\left(\bmod \hat{m}^{h-i} d \mathbb{Z}\right)$, as desired.

Proof of (2). By construction, we have

$$
s_{i-1}(n) \cdot n=s_{i}(n) \cdot m+r_{i}(n)
$$

and

$$
s_{i-1}\left(n^{\prime}\right) \cdot n^{\prime}=s_{i}\left(n^{\prime}\right) \cdot m+r_{i}\left(n^{\prime}\right)
$$

for all $i \in\{1, \ldots, h\}$. By hypothesis (2), we get $s_{i}(n)-s_{i}\left(n^{\prime}\right)=\left(s_{i-1}(n) \cdot n-\right.$ $\left.s_{i-1}\left(n^{\prime}\right) \cdot n^{\prime}\right) / m$, which we write in the form

$$
s_{i}(n)-s_{i}\left(n^{\prime}\right)=\frac{\left(s_{i-1}(n)-s_{i-1}\left(n^{\prime}\right)\right) \cdot \hat{n}}{\hat{m}}+\frac{s_{i-1}\left(\hat{n}^{\prime}\right)\left(\hat{n}-\hat{n}^{\prime}\right)}{\hat{m}},
$$

where $\hat{n}=n / d$ and $\hat{n}^{\prime}=n^{\prime} / d$. We show by induction on $k$ that $\hat{n} \equiv \hat{n}^{\prime}\left(\bmod \hat{m}^{k} \mathbb{Z}\right)$ for $k=1, \ldots, h$. The case $k=1$ follows directly from equation (2) for $i=1$ (recall that $s_{0}(n)=1=s_{0}\left(n^{\prime}\right)$ ). For the inductive step, we assume that $2 \leq k \leq h$ et $\hat{n} \equiv \hat{n}^{\prime}\left(\bmod \hat{m}^{k-1} \mathbb{Z}\right)$, which implies that the second term on the right-hand side of equation (2) is a multiple of $\hat{m}^{k-2}$ (it is an integer in particular). We then proceed in $k$ steps using equation (2) and the fact that $\hat{m}$ and $\hat{n}$ are coprime:

- for $i=k$ we get $s_{k-1}(n) \equiv s_{k-1}\left(n^{\prime}\right)(\bmod \hat{m} \mathbb{Z})$;

- for $i=k-1($ if $k \geq 3)$ we get $s_{k-2}(n) \equiv s_{k-2}\left(n^{\prime}\right)\left(\bmod \hat{m}^{2} \mathbb{Z}\right)$;

- for $i=k-2, \ldots, 2$ we get the sequence of congruences

$$
s_{k-3}(n) \equiv s_{k-3}\left(n^{\prime}\right) \quad\left(\bmod \hat{m}^{3} \mathbb{Z}\right), \ldots, s_{1}(n) \equiv s_{1}\left(n^{\prime}\right) \quad\left(\bmod \hat{m}^{k-1} \mathbb{Z}\right) ;
$$

- for $i=1$ we get $\hat{n} \equiv \hat{n}^{\prime}\left(\bmod \hat{m}^{k} \mathbb{Z}\right)$.

Finally, we obtain $n \equiv n^{\prime}\left(\bmod \hat{m}^{k} d \mathbb{Z}\right)$, which completes the proof.

Proposition 2.6. (i) The functions $r_{i}$ and $s_{i}$ admit unique continuous extensions

$$
r_{i}: \mathbb{Z}_{m} \rightarrow\{0, \ldots,|m|-1\}, \quad s_{i}: \mathbb{Z}_{m} \rightarrow \mathbb{Z}_{m}
$$

such that

$$
r_{0}(\xi)=0, \quad s_{0}(\xi)=1
$$

$$
\xi s_{i-1}(\xi)=m s_{i}(\xi)+r_{i}(\xi) \text { with } r_{i}(\xi) \in\{0, \ldots,|m|-1\} \text { for every } i \geq 1 \text {. }
$$

Let $\xi, \xi^{\prime} \in \mathbb{Z}_{m}$ such that $\operatorname{gcd}(\xi, m)=\operatorname{gcd}\left(\xi^{\prime}, m\right)=d$ and let $h \in \mathbb{N} \backslash\{0\}$. Setting $\hat{m}=m / d$, the following statements are equivalent: 
(1) $\xi \equiv \xi^{\prime}\left(\bmod \hat{m}^{h} d \mathbb{Z}_{m}\right)$ holds;

(2) $r_{i}(\xi)=r_{i}\left(\xi^{\prime}\right)$ for $i=1, \ldots, h$.

(ii) Let $\xi, \xi^{\prime} \in \mathbb{Z}_{m}$. The following statements are equivalent:

(1) There is some $d \in \mathbb{N}$ such that $\operatorname{gcd}(\xi, m)=\operatorname{gcd}\left(\xi^{\prime}, m\right)=d$ and $\pi(\xi / d)=$ $\pi\left(\xi^{\prime} / d\right)$ where $\pi: \mathbb{Z}_{m} \rightarrow \mathbb{Z}_{m / d}$ is the canonical ring homomorphism;

(2) $r_{i}(\xi)=r_{i}\left(\xi^{\prime}\right)$ for every $i$.

(iii) The mapping $\xi \mapsto\left(r_{i}(\xi)\right)_{i \geq 1}$ defines a homeomorphism from $\mathbb{Z}_{m}^{\times}$onto $(\mathbb{Z} / m \mathbb{Z})^{\times} \times(\mathbb{Z} / m \mathbb{Z})^{\mathbb{N}}$ endowed with its product topology. (Here we identify $\mathbb{Z} / m \mathbb{Z}$ with the set $\{0, \ldots,|m|-1\}$ and $(\mathbb{Z} / m \mathbb{Z})^{\times}$with the set of integers $k \in\{0, \ldots,|m|-1\}$ coprime with $\mathrm{m}$.)

Proof. (i) Proposition 2.5 shows that the functions $r_{i}, s_{i}$ are uniformly continuous with respect to the $m$-adic topology on $\mathbb{Z}$ and that $r_{i}$ is moreover constant on $m$-adic balls of radius $|m|^{-i}$. The existence and uniqueness of the extensions to $\mathbb{Z}_{m}$ follow. The inductive formulas of Definition 2.4 extend continuously on $\mathbb{Z}_{m}$, which gives $\left(1_{\xi}\right)$ and $\left(2_{\xi}\right)$. Let us now pick $n, n^{\prime} \in \mathbb{Z}$ such that $n \equiv \xi$ and $n^{\prime} \equiv \xi^{\prime}\left(\bmod m^{h} \mathbb{Z}_{m}\right)$. Then, one has $n \equiv n^{\prime}\left(\bmod \hat{m}^{h} d\right) \mathbb{Z}$ if and only if $\xi \equiv \xi^{\prime}\left(\bmod \hat{m}^{h} d \mathbb{Z}_{m}\right)$. As $r_{i}$ is constant on $m$-adic balls of radius $|m|^{-i}$ we have $r_{i}(n)=r_{i}(\xi)$ and $r_{i}\left(n^{\prime}\right)=r_{i}\left(\xi^{\prime}\right)$ for $i=1, \ldots, h$. The equivalence between (1) and (2) now follows readily from Proposition 2.5.

(ii) As $\operatorname{gcd}(m, \xi)=\operatorname{gcd}\left(m, r_{1}(\xi)\right)$ for every $\xi \in \mathbb{Z}_{m}$ by $\left(2_{\xi}\right)$, the result immediately follows from (i).

(iii) Let $\xi \in \mathbb{Z}_{m}^{\times}$. By $\left(2_{\xi}\right)$, we have $r_{1}(\xi) \in(\mathbb{Z} / m \mathbb{Z})^{\times}$. For any $\xi, \xi^{\prime} \in \mathbb{Z}_{m}^{\times}$, we have $d=\operatorname{gcd}(\xi, m)=\operatorname{gcd}\left(\xi^{\prime}, m\right)=1$. We deduce from (2) that the map $R: \xi \mapsto\left(r_{i}(\xi)\right)_{i \geq 1}$ defines a continuous embedding from $\mathbb{Z}_{m}^{\times}$into $P:=(\mathbb{Z} / m \mathbb{Z})^{\times} \times$ $(\mathbb{Z} / m \mathbb{Z})^{\mathbb{N}}$. As $\mathbb{Z}_{m}^{\times}$and $P$ are compact, it suffices to show that $R$ has a dense image. Let $E_{h}$ be the set of integers $k \in\left\{0, \ldots,|m|^{h}-1\right\}$ coprime with $m$. Consider the map $R_{h}: \xi \mapsto\left(r_{i}(\xi)\right)_{1 \leq i \leq h}$. This is an injective map from $E_{h}$ into $P_{h}:=$ $(\mathbb{Z} / m \mathbb{Z})^{\times} \times(\mathbb{Z} / m \mathbb{Z})^{h-1}$ by the equivalence of (1) and (2). As $E_{h}$ and $P_{h}$ have both cardinality $\varphi(m)|m|^{h-1}$, where $\varphi$ denotes the Euler function, the map $R_{h}$ is a bijection. Hence $R$ maps the set of integers coprime with $|m|$ onto a dense subset of $P$.

The following proposition shows that the restriction of $s_{i}$ to a suitable congruence class is a polynomial in $n$ with coefficients in $\mathbb{Q}$.

Proposition 2.7. Let $\xi \in \mathbb{Z}_{m}$. We define recursively $P_{h}(X)=P_{h, \xi}(X)$ by

$$
P_{0}(X)=m \quad \text { and } \quad P_{h}(X)=X P_{h-1}(X)-r_{h}(\xi) \quad \text { for } h \geq 1 .
$$

Then we have:

(i) $P_{h}(X)=m X^{h}-r_{1}(\xi) X^{h-1}-\cdots-r_{h}(\xi) \in \mathbb{Z}[X]$. 
(ii) $s_{h}(n)=\frac{1}{m} P_{h}\left(\frac{n}{m}\right)$ for all $h \geq 0$ and all $n \in \mathbb{Z}$ such that $n \equiv \xi\left(\bmod \hat{m}^{h} d \mathbb{Z}_{m}\right)$.

Proof. (i) The proof is an obvious induction on $h$.

(ii) First, observe that $r_{i}(n)=r_{i}(\xi)$ for all $i \leq h$ and all $n \in \mathbb{Z}$ such that $n \equiv \xi$ $\left(\bmod \hat{m}^{h} d \mathbb{Z}_{m}\right)$ by Proposition 2.6. An easy induction on $h$ using the definitions of $s_{i}(n)$ and $P_{i}(X)$ gives the conclusion.

2.2. Stabilizers. This section is devoted to the study of the stabilizers $B, B_{m}$ and $B_{\xi}$.

Let $b_{0}=b, b_{1}=a b^{m} a^{-1}, b_{i}=a b_{i-1} b_{0}^{-r_{i-1}(\xi)} a^{-1}$ for $i \geq 2$. The following lemma shows that $b_{i}$ defines an element of $B$ for every $i$.

Lemma 2.8. Let $i \geq 1$. We have $b_{i}=b^{\frac{\xi n}{m} P_{i-1}\left(\frac{\xi_{n}}{m}\right)}$ in $\mathrm{BS}\left(m, \xi_{n}\right)$ for all $n$ large enough.

Proof. We show by induction on $i$ that:

for every $i \geq 1$ we have $b_{i}=b^{\xi_{n} s_{i-1}\left(\xi_{n}\right)}$ in $\operatorname{BS}\left(m, \xi_{n}\right)$ for all $n$ large enough. (3)

Since $b_{1}=a b^{m} a^{-1}=b^{\xi_{n}}$ in $\operatorname{BS}\left(m, \xi_{n}\right)$ and $s_{0}\left(\xi_{n}\right)=1$ for all $n$, (3) holds if $i=1$. Assume (3) holds for some $i \geq 1$. By the induction hypothesis we have $b_{i+1}=a b_{i} b^{-r_{i}(\xi)} a^{-1}=a b^{\xi_{n} s_{i-1}\left(\xi_{n}\right)-r_{i}(\xi)} a^{-1}$ for all $n$ large enough. Recall that $\xi_{n}$ tends to $\xi$ in $\mathbb{Z}_{m}$ and hence $r_{i}\left(\xi_{n}\right)=r_{i}(\xi)$ for all $n$ large enough by Proposition 2.6. By Definition 2.4, we obtain $b_{i+1}=b^{\xi_{n} s_{i}\left(\xi_{n}\right)}$ in $\mathrm{BS}\left(m, \xi_{n}\right)$ for all $n$ large enough. Since $\xi_{n} s_{i}\left(\xi_{n}\right)=\frac{\xi_{n}}{m} P_{i}\left(\frac{\xi_{n}}{m}\right)$ for all $n$ large enough by Proposition 2.7 , the proof is then complete.

We can generalize the previous lemma by assigning to every $\gamma \in B$ a polynomial with integer coefficients.

Proposition 2.9. (i) Let $w \in \mathbb{F}_{2}$ with image $\gamma \in B$. Then there is a unique polynomial $P_{\gamma}(X) \in \mathbb{Z}[X]$ independent of $w$ such that $w=b^{P_{\gamma}\left(\xi_{n} / m\right)}$ in $\operatorname{BS}\left(m, \xi_{n}\right)$ for all $n$ large enough.

(ii) The map $q: \gamma \mapsto P_{\gamma}(X)$ defines an injective homomorphism from $B$ into $\mathbb{Z}[X]$. The abelian group $\mathfrak{B}=q(B)$ is freely generated by $\left\{1, X P_{0}(X), X P_{1}(X)\right.$, $\left.X P_{2}(X), \ldots\right\}$. Hence $B$ is freely generated by the set $\left\{b_{0}, b_{1}, b_{2}, b_{3}, \ldots\right\}$.

(iii) Let $w \in \mathbb{F}_{2}$ with image $\gamma \in B$. Then $\gamma$ belongs to $B_{m}$ if and only if

$$
P_{\gamma}(X)=k_{0}+k_{1} m X+k_{2} X P_{1}(X)+\cdots+k_{t} X P_{t-1}(X)
$$

with $k_{0}+k_{1} r_{1}(\xi)+k_{2} r_{2}(\xi)+\cdots+k_{t} r_{t}(\xi) \equiv 0(\bmod m \mathbb{Z})$. Moreover the abelian group $\mathfrak{B}_{m}=q\left(B_{m}\right)$ is freely generated by $P_{0}(X)=m, P_{1}(X)=X P_{0}(X)-r_{1}(\xi)$, $P_{2}(X)=X P_{1}(X)-r_{2}(\xi), \ldots$. Hence $B_{m}$ is freely generated by $\left\{b_{0}^{m}, b_{1} b_{0}^{-r_{1}(\xi)}\right.$, $\left.b_{2} b_{0}^{-r_{2}(\xi)}, \ldots\right\}$. 
(iv) The abelian group $\mathfrak{B}_{\xi}=q\left(B_{\xi}\right)$ is freely generated by $\left\{X P_{0}(X), X P_{1}(X)\right.$, $\left.X P_{2}(X), \ldots\right\}$. The abelian group $B_{\xi}$ is freely generated by $\left\{b_{1}, b_{2}, b_{3}, \ldots\right\}$.

(v) For any $\gamma \in B_{m}$, we have $q\left(a \gamma a^{-1}\right)=X P_{\gamma}(X)$. Moreover the map $a \mapsto$ $(0,1), b \mapsto(1,0)$ induces a surjective homomorphism $q_{m, \xi}: \overline{\mathrm{BS}}(m, \xi) \rightarrow \mathbb{Z} \imath \mathbb{Z}=$ $\mathbb{Z}\left[X^{ \pm 1}\right] \rtimes \mathbb{Z}$ whose restriction to $B$ coincides with $q$.

(vi) Let $d=\operatorname{gcd}(m, \xi), \hat{m}=m / d$, and let $\pi: \mathbb{Z} \rightarrow \mathbb{Z}_{\hat{m}}$ be the canonical map. The maps $\chi: \gamma \mapsto P_{\gamma}(\xi / m)$ and $\hat{\chi}=\pi \circ \chi$ define homomorphisms from $B$ to $\mathbb{Z}_{m}$ and $\mathbb{Z}_{\hat{m}}$, respectively. Their kernels satisfy: $\operatorname{ker} \chi \subset \bigcap_{i \geq 0} a^{-i} B a^{i} \subset \operatorname{ker} \hat{\chi}$. Moreover, if $\bigcap_{i \geq 0} a^{-i} B a^{i}$ is non-trivial, then it contains some $\gamma \in B_{m} \backslash B_{\xi}$.

(vii) Let $\mathfrak{C}$ be the image of $\mathfrak{B}_{\xi}$ by the map $\iota: P(X) \mapsto \frac{X-1}{X} P(X)$. Then $\mathfrak{C}$ is a subgroup of $\mathfrak{B}$ and $\mathfrak{B} / \mathfrak{C}$ is an infinite cyclic group generated by the image of 1 .

Proof. (i) The uniqueness of $P_{\gamma}(X)$ follows from the fact that a non-zero polynomial with coefficients in $\mathbb{Q}$ has only finitely many zeros in $\mathbb{Q}$. Using the fact that BS $\left(m, \xi_{n}\right)$ converges to $\overline{\mathrm{BS}}(m, \xi)$, we also deduce that $P_{\gamma}(X)$ is independent of $w$. To show the existence of $P_{\gamma}(X)$ we write $w=b^{e_{0}} a^{\varepsilon_{1}} b^{e_{1}} \ldots a^{\varepsilon_{h}} b^{e_{h}}$ with $\varepsilon_{j}= \pm 1$ for $j=1, \ldots, h$. By Lemma 2.2, $w$ reduces to a power of $b$ in $\operatorname{BS}\left(m, \xi_{n}\right)$ for all $n$ large enough. Since the powers of $a$ must eventually cancel out, we have $h=2 t$ for some $t \in \mathbb{N}$. By [GS08], Lemma 2.6 with $d=1$, there exist $k_{0}, \ldots, k_{t} \in \mathbb{Z}$ depending only on $w$ and $\xi$ such that $w=b^{\alpha(n)}$ in $\operatorname{BS}\left(m, \xi_{n}\right)$ for all $n$ such that $\xi_{n} \equiv \xi\left(\bmod m^{t} \mathbb{Z}_{m}\right)$ with $\left|\xi_{n}\right|$ large enough and

$$
\alpha(n)=k_{0}+k_{1} \xi_{n}+k_{2} s_{1}\left(\xi_{n}\right) \xi_{n}+\cdots+k_{t} s_{t-1}\left(\xi_{n}\right) \xi_{n},
$$

the latter equation being Formula $\left(^{*}\right)$ in the proof of [GS08], Lemma 2.6. Since $\xi_{n}$ tends to $\xi$ in $\mathbb{Z}_{m}$ and $\left|\xi_{n}\right|$ tends to infinity as $n$ goes to infinity, the equality (4) holds for all $n$ large enough. Using Proposition 2.7, we can write $\alpha(n)=P_{\gamma}\left(\frac{\xi_{n}}{m}\right)$ with

$$
P_{\gamma}(X)=k_{0}+k_{1} X P_{0}(X)+k_{2} X P_{1}(X)+\cdots+k_{t} X P_{t-1}(X) .
$$

(ii) The map $q: \gamma \mapsto P_{\gamma}(X)$ trivially induces a homomorphism from $B$ to $\mathbb{Z}[X]$ such that $q(b)=1$. Let $w \in \mathbb{F}_{2}$ with image $\gamma \in B$. If $P_{\gamma}(X)$ is the zero polynomial, then $w$ is trivial in $\operatorname{BS}\left(m, \xi_{n}\right)$ for all $n$ large enough and hence it is trivial in $\overline{\mathrm{BS}}(m, \xi)$. Thus $q$ is injective. It is immediate from (5) that $q(B)$ is a subgroup of the free abelian group with basis $\left\{1, X P_{0}(X), X P_{1}(X), \ldots\right\}$. It follows from Lemma 2.8 that $q\left(b_{i}\right)=X P_{i-1}(X)$ for all $i \geq 1$, so that $q(B)$ coincides with the latter group.

(iii) Let $w \in \mathbb{F}_{2}$ with image $\gamma \in B$. By (i) we can write $w=b^{P_{\gamma}\left(\xi_{n} / m\right)}$ in $\operatorname{BS}\left(m, \xi_{n}\right)$ for all $n$ large enough. Since $\xi_{n} s_{i-1}\left(\xi_{n}\right) \equiv r_{i}(\xi)(\bmod m \mathbb{Z})$ for every $i \geq 1$ and for all $n$ large enough, we deduce from (5) that $P_{\gamma}\left(\xi_{n} / m\right) \equiv$ $k_{0}+k_{1} r_{1}(\xi)+k_{2} r_{2}(\xi)+\cdots+k_{t} r_{t}(\xi)(\bmod m \mathbb{Z})$ for all $n$ large enough. By Lemma 2.2, we have: $\gamma \in B_{m}$ if and only if $\alpha(n) \equiv 0(\bmod m \mathbb{Z})$. This proves the first claim. We easily deduce that $\left\{m, X P_{0}(X)-r_{1}(\xi), X P_{1}(X)-r_{2}(\xi), \ldots\right\}$ freely generates $\mathfrak{B}_{m}$. Since $q\left(b_{0}^{m}\right)=m$ and $q\left(b_{i} b_{0}^{-r_{i}(\xi)}\right)=X P_{i-1}(X)-r_{i}(\xi)$ the set $\left\{b_{0}^{m}, b_{1} b_{0}^{-r_{1}(\xi)}, b_{2} b_{0}^{-r_{2}(\xi)}, \ldots\right\}$ freely generates $B_{m}$. 
(iv) Since $B_{\xi}=a B_{m} a^{-1}$, we deduce from (iii) and the definition of $b_{i}$ that $\left\{b_{1}, b_{2}, \ldots\right\}$ generates $B_{\xi}$. As the elements $q\left(b_{i}\right)=X P_{i-1}(X)(i \geq 1)$ freely generate $\mathfrak{B}_{\xi}$, we deduce that $\left\{b_{1}, b_{2}, \ldots\right\}$ freely generates $B_{\xi}$.

(v) Let $w \in \mathbb{F}_{2}$ with image $\gamma \in B_{m}$. We can write $w=b^{P_{\gamma}\left(\xi_{n} / m\right)}$ in BS $\left(m, \xi_{n}\right)$ for all $n$ large enough with $P_{\gamma}\left(\xi_{n} / m\right) \equiv 0(\bmod m \mathbb{Z})$. Thus $a \gamma a^{-1}=b^{\left(\xi_{n} / m\right) P_{\gamma}\left(\xi_{n} / m\right)}$ in BS $\left(m, \xi_{n}\right)$ for all $n$ large enough. Hence $q\left(a \gamma a^{-1}\right)=X P_{\gamma}(X)$ by definition of $q$.

The map $q_{m, \xi}$ is a well-defined homomorphism by [GS08], Theorem 3.12. Using the first part of (v), we easily deduce by induction that $q\left(b_{i}\right)=q_{m, \xi}\left(b_{i}\right)$ for every $i \geq 0$, which completes the proof.

(vi) The map $\gamma \mapsto P_{\gamma}(\xi / m)$ is a well-defined homomorphism from $B$ to $\mathbb{Z}^{-1} \mathbb{Z}_{m}$. By Proposition 2.6 we have $s_{i}(\xi) \in \mathbb{Z}_{m}$ for every $i$. By Proposition 2.7, we have $\frac{1}{m} P_{i}\left(\frac{\xi n}{m}\right)=s_{i}\left(\xi_{n}\right) \in \mathbb{Z}$ for every $n$ large enough and hence $\frac{1}{m} P_{i}\left(\frac{\xi}{m}\right)=s_{i}(\xi) \in \mathbb{Z}_{m}$ by Proposition 2.6. Now it follows from (ii) that $P_{\gamma}(\xi / m)$ belongs to $\mathbb{Z}_{m}$ for every $\gamma \in B$.

Let $\gamma \in \operatorname{ker} \chi$ and let $i \geq 0$. For all $n$ large enough $m^{i}$ divides $P_{\gamma}\left(\xi_{n} / m\right)$. We deduce from (i) and Lemma 2.2 that $a^{i} \gamma a^{-i} \in B$. Thus $\gamma \in \bigcap_{i \geq 0} a^{-i} B a^{i}$.

Let $\eta=\pi(\xi / d)$. By $(v)$ we have $\hat{\chi}\left(a \gamma a^{-1}\right)=\frac{\eta}{\hat{m}} \hat{\chi}(\gamma) \in \mathbb{Z}_{\hat{m}}$ for every $\gamma \in B \cap$ $a^{-1} B a$. Consider now $\gamma \in B$ such that $a^{i} \gamma a^{-i} \in B$ for every $i$. As $\operatorname{gcd}(\hat{m}, \eta)=1$, $\hat{m}^{i}$ divides $\hat{\chi}(\gamma)$ for every $i$. Therefore we have $\gamma \in \operatorname{ker} \hat{\chi}$.

Assume that $B^{\prime}=\bigcap_{i \geq 0} a^{-i} B a^{i}$ is non-trivial. Clearly $B^{\prime} \subset B_{m}$ and any $\gamma \in B^{\prime}$ such that $P_{\gamma}(X)$ has minimal degree does not belong to $B_{\xi}$ by $(v)$.

(vii) It follows from the definition of $P_{i}(X)$ that multiplication by $\frac{X-1}{X}$ maps $X P_{0}(X)$ to $-m+X P_{0}(X)$ and $X P_{i}(X)$ to $r_{i}(\xi)-X P_{i-1}(X)+X P_{i}(X)$ for every $i \geq 1$. Therefore $\mathfrak{C}$ is a subgroup of $\mathfrak{B}$. A straightforward induction on $i$ shows that the image of $X P_{i}(X)$ in $\mathfrak{B} / \mathfrak{C}$ lies inside the cyclic subgroup generated by the image of 1 . As $\mathfrak{B}_{\xi}$ does not contain any constant polynomial, neither does $\mathfrak{C}$. Therefore $\mathfrak{B} / \mathfrak{C}$ is infinite.

Now we can give a simple description of the HNN structure of $\overline{\mathrm{BS}}(m, \xi)$. Let $\widetilde{\mathrm{BS}}(m, \xi)$ be the HNN extension of basis $E$ with conjugated subgroups $E_{m, \xi}$ and $E_{1}$ stable letter $a$, where $E, E_{m}, E_{\xi}$ are free abelian groups of countable rank, namely

$$
\begin{aligned}
E & =\mathbb{Z} e_{0} \oplus \mathbb{Z} e_{1} \oplus \mathbb{Z} e_{2} \oplus \cdots, \\
E_{m, \xi} & =\mathbb{Z} m e_{0} \oplus \mathbb{Z}\left(e_{1}-r_{1}(\xi) e_{0}\right) \oplus \mathbb{Z}\left(e_{2}-r_{2}(\xi) e_{0}\right) \oplus \cdots, \\
E_{1} & =\mathbb{Z} e_{1} \oplus \mathbb{Z} e_{2} \oplus \cdots,
\end{aligned}
$$

and where conjugacy from $E_{m, \xi}$ to $E_{1}$ is defined by $a\left(m e_{0}\right) a^{-1}=e_{1}$ and $a\left(e_{i}-r_{i}(\xi) e_{0}\right) a^{-1}=e_{i+1}$. We denote by $\phi$ the isomorphism from $E_{m, \xi}$ to $E_{1}$ induced by $\tau_{a}$.

Corollary 2.10. The map defined by $f(a)=a$ and $f(b)=e_{0}$ induces an isomorphism from $\overline{\mathrm{BS}}(m, \xi)$ to $\widetilde{\mathrm{BS}}(m, \xi)$. The inverse map is determined by $f^{-1}(a)=a$ and $f^{-1}\left(e_{i}\right)=b_{i}$ for every $i \geq 0$. 
Proof. This is a straightforward corollary of Propositions 2.1 and 2.9.

We then have three different notations for the same group, namely

- $\overline{\mathrm{BS}}(m, \xi)=\operatorname{HNN}\left(B, B_{m}, B_{\xi}, \tau\right)$ where $B$ is generated by $b=b_{0}, b_{1}, b_{2}, \ldots$,

- $\widetilde{\mathrm{BS}}(m, \xi)=\operatorname{HNN}\left(E, E_{m, \xi}, E_{1}, \phi\right)$ where $E$ is generated by $e_{0}, e_{1}, e_{2}, \ldots$,

- $\mathfrak{B} \subseteq(m, \xi)=\operatorname{HNN}\left(\mathfrak{B}, \mathfrak{B}_{m}, \mathfrak{B}_{\xi}, \theta\right)$ where $\mathfrak{B}$ is generated by $1, X P_{0}(X)$, $X P_{1}(X), \ldots$ and $\theta$ is defined in the obvious way.

Our favoured HNN extension is $\widetilde{\mathrm{BS}}(m, \xi)$ since it considerably simplifies notations when different parameters $m$ and $\xi$ are considered (Section 5 and Section 6). Nevertheless $\overline{\mathrm{BS}}(m, \xi)$ is useful when we still need to see this group as limit in the space of marked groups (Section 5) and the HNN extension $\mathfrak{B} \subseteq(m, \xi)$ involving polynomials is advantageous to study relations in the group (Section 5).

\section{An infinite presentation built up from $\xi$}

In this section we use of the HNN decomposition of $\overline{\mathrm{BS}}(m, \xi)$ to give an infinite group presentation which depends explicitly on $m$ and the sequence $\left(r_{i}(\xi)\right)$. A group presentation $\langle X \mid R\rangle$ is said to be minimal if the kernel of the natural homomorphism $\left\langle X \mid R^{\prime}\right\rangle \rightarrow\langle X \mid R\rangle$ is non-trivial for all $R^{\prime} \varsubsetneqq R$.

Theorem 3.1. The group $\overline{\mathrm{BS}}(m, \xi)$ admits the following infinite presentation:

$$
\left\langle a, b \mid\left[b, b_{i}\right]=1, i \geq 1\right\rangle
$$

with $b_{1}=a b^{m} a^{-1}$ and $b_{i}=a b_{i-1} b^{-r_{i-1}(\xi)} a^{-1}$ for every $i \geq 2$.

In particular, we have $\overline{\mathrm{BS}}(m, \xi)(m, 0)=\left\langle a, b \mid\left[b, a^{i} b^{m} a^{-i}\right]=1, i \geq 1\right\rangle$. The latter presentation is moreover minimal.

In the case $\xi \neq 0$, we previously showed ([GS08], Theorem 4.1) that $\overline{\mathrm{BS}}(m, \xi)$ cannot be finitely presented. The following consequence is therefore immediate.

Corollary 3.2. No group $\overline{\mathrm{BS}}(m, \xi)$ is finitely presented.

Proof of Theorem 3.1. We divide the proof into two parts:

(1) We show that $\overline{\mathrm{BS}}(m, \xi)=\left\langle a, b \mid\left[b, b_{i}\right]=1, i \geq 1\right\rangle$ using Propositions 2.1 and 2.9.

(2) We show that this presentation is minimal in the case $\xi=0$ using basic facts on graph groups.

Proof of (1). By Proposition 2.9 we can define an isomorphism $\phi: B_{m} \rightarrow B_{\xi}$ by $\phi\left(b_{0}^{m}\right)=b_{1}, \phi\left(b_{i} b_{0}^{-d r_{i}(\xi)}\right)=b_{i+1}$ for $i \geq 1$. Making use of the HNN decomposition of Proposition 2.1, we deduce that $\overline{\mathrm{BS}}(m, \xi)$ admits the presentation 
$\left\langle a, B \mid a \gamma a^{-1}=\phi(\gamma), \gamma \in B_{m}\right\rangle$. Since $a$ and $b$ generate $\overline{\mathrm{BS}}(m, \xi)$ and $B$ is a free abelian group (Proposition 2.9), we deduce that

$$
\overline{\mathrm{BS}}(m, \xi)=\left\langle a, b \mid\left[b_{i}, b_{j}\right]=1,0 \leq i<j\right\rangle
$$

(Note that the relations $a b_{0}^{m} a^{-1}=\phi\left(b_{0}^{m}\right)$ and $a b_{i} b_{0}^{-r_{i}(\xi)} a^{-1}=\phi\left(b_{i} b_{0}^{-r_{i}(\xi)}\right.$ ) are satisfied by definition of $b_{i}$.) We show by induction on $i \geq 0$ the following claim: any relation $\left[b_{i}, b_{j}\right]=1$ with $0 \leq i<j$ is a consequence of relations $\left[b_{0}, b_{k}\right]=1$ with $1 \leq k \leq j$. The case $i=0$ is trivial. Assume now that $i \geq 1$. Using definitions, we can write $\left[b_{i}, b_{j}\right]=a\left[b_{i-1} b_{0}^{-r_{i-1}(\xi)}, b_{j-1} b_{0}^{-r_{j-1}(\xi)}\right] a^{-1}$. By induction hypothesis the relation $\left[b_{i-1}, b_{j-1}\right]=1$ is a consequence of relations $\left[b_{0}, b_{k}\right]=1$ with $1 \leq k \leq$ $j-1$. Hence $\left[b_{i}, b_{j}\right]=1$ is a consequence of relations $\left[b_{0}, b_{k}\right]=1$ with $1 \leq k \leq j$. The induction is then complete. Thus all relations $\left[b_{i}, b_{j}\right]=1$ with $1 \leq i<j$ can be deleted in presentation (6).

Proof of (2). It remains to show that $\overline{\mathrm{BS}}(m, \xi)=\left\langle a, b \mid\left[b_{0}, b_{i}\right]=1, i \geq 1\right\rangle$ is a minimal presentation when $\xi=\pi(\xi)=0$. In this case we have $r_{i}(\xi)=0$, and hence $b_{i}=a^{i} b^{m} a^{-i}$ for all $i \geq 1$. We fix some $k \geq 1$ and we show that $\left[b_{0}, b_{k}\right]=1$ does not hold in the group $G_{k}:=\left\langle a, b \mid\left[b_{0}, b_{i}\right]=1, k \neq i \geq 1\right\rangle$.

To prove this we consider the group $\widetilde{B}=\left\langle g_{0}, g_{1}, \ldots\left|\left[g_{i}, g_{j}\right]=1,\right| i-j \mid \neq k\right\rangle$ and its subgroups $\widetilde{B}_{m}=\left\langle g_{0}^{m}, g_{1}, g_{2}, \ldots\right\rangle$ and $\widetilde{B}_{\xi}=\left\langle g_{1}, g_{2}, \ldots\right\rangle$. We will use basic facts on graph groups, i.e., groups defined by a presentation whose relators are commutators of some pairs of the generators. (Such presentations are often encoded by a graph whose vertices correspond to the generators and whose edges tell which ones commute; this explains the terminology.) Graph groups are also referred to as right-angled Artin groups or partially commutative groups. Let $G=\langle X \mid R\rangle$ be a graph group. Any element of $G$ can be written as a word $c_{1} c_{2} \ldots c_{l}$ where each syllable $c_{i}$ belongs to some cyclic group generated by some element of $X$. We consider three types of moves that we can perform on such words.

1. Remove a syllable $c_{i}=1$.

2. Replace consecutive syllables $c_{i}$ and $c_{i+1}$ in the same cyclic subgroup by the single syllable $\left(c_{i} c_{i+1}\right)$.

3. For consecutive syllables $c_{i} \in\langle x\rangle$ and $c_{i+1} \in\left\langle x^{\prime}\right\rangle$ with $x, x^{\prime} \in X,\left[x, x^{\prime}\right] \in R$, exchange $c_{i}$ and $c_{i+1}$.

If $g \in G$ is represented by a word $w=c_{1} \ldots c_{l}$ which cannot be changed to a shorter word using any sequence of the above moves, then $w$ is said to be a normal form for $g$. We will use the following results:

Theorem 3.3 ([Bau81]). (Normal Form Theorem) A normal form in a graph group represents the trivial element if and only if it is the trivial word.

(Abelian Subgroups) Any abelian subgroup of a graph group is a free abelian group. 
We define the partial map $\psi: \widetilde{B}_{\xi} \rightarrow \widetilde{B}_{m}$ by $\psi\left(g_{1}\right)=g_{0}^{m}$ and $\psi\left(g_{i}\right)=g_{i-1}$ for every $i \geq 2$. By using the Normal Form Theorem, we can readily show that $\widetilde{B}_{\xi}=\left\langle g_{1}, g_{2}, \ldots\left|\left[g_{i}, g_{j}\right]=1,\right| i-j \mid \neq k\right\rangle$. We clearly have $\left[\psi\left(g_{i}\right), \psi\left(g_{j}\right)\right]=1$ in $\widetilde{B}_{m}$ for any $i, j \geq 1$ such that $|i-j| \neq k$. Hence $\psi$ induces a surjective homomorphism from $\widetilde{B}_{\xi}$ onto $\widetilde{B}_{m}$.

Let $g \in \widetilde{B}_{\xi}$. Replacing every $g_{i}$ by $\psi\left(g_{i}\right)$ in any non-trivial normal form for $g$ in $\widetilde{B}$ clearly leads to a non-trivial normal form for $\psi(g)$ in $\widetilde{B}$. Hence $\psi$ is injective, which proves that $\psi$ is an isomorphism. Let $\widetilde{\phi}$ be its inverse homomorphism. We set $\widetilde{G}_{k}:=\left\langle\widetilde{B}, a \mid a g a^{-1}=\widetilde{\phi}(g), g \in \widetilde{B}_{m}\right\rangle$. We trivially check that the map $a \mapsto a$, $b \mapsto g_{0}$ induces a surjective homomorphism from $G_{k}$ onto $\widetilde{G}_{k}$ that maps $\left[b_{0}, b_{k}\right]$ to $\left[g_{0}, g_{k}\right]$. Since the graph group $\widetilde{B}$ embeds isomorphically into the HNN extension $\widetilde{G}_{k}$, we have $\left[g_{0}, g_{k}\right] \neq 1$ and hence $\left[b_{0}, b_{k}\right] \neq 1$.

\section{4. $C^{*}$-simplicity}

We first recall some definitions. Let $\Gamma=\overline{\mathrm{BS}}(m, \xi)$ and let $\sigma: \overline{\mathrm{BS}}(m, \xi) \rightarrow \mathbb{Z}$ be the surjective homomorphism defined by $\sigma(a)=1$ and $\sigma(b)=0$. Let $\mathbb{Z}$ ? $\mathbb{Z}=\mathbb{Z}\left[X^{ \pm 1}\right] \rtimes_{X} \mathbb{Z}$ where the action of $\mathbb{Z}$ on the additive group of $\mathbb{Z}\left[X^{ \pm 1}\right]$ is the multiplication by $X$. This group is generated by $\{(1,0),(0,1)\}$ and the map $a \mapsto(0,1), b \mapsto(1,0)$ induces a surjective homomorphism $q_{m, \xi}$ from $\Gamma$ onto $\mathbb{Z} \imath \mathbb{Z}$ [GS08], Theorem 3.12. By (v) and (vi) of Proposition 2.9, the restriction of $q_{m, \xi}$ to $B$ (which identifies with a subgroup of $\mathbb{Z}[X]$ ) is the identity and the map $\gamma \mapsto P_{\gamma}(\xi / m)$ is a well-defined homomorphism from $B$ to the additive group of $\mathbb{Z}_{m}$.

Given a group $\Gamma$, recall that its reduced $C^{*}$-algebra $C_{r}^{*}(\Gamma)$ is the closure for the operator norm of the group algebra $\mathbb{C}[\Gamma]$ acting by the left-regular representation on the Hilbert space $\ell^{2}(\Gamma)$. For an introduction to group $\mathrm{C}^{*}$-algebras, see for example [Dav96], Chapter VII. A group is $\mathrm{C}^{*}$-simple if it is infinite and if its reduced $\mathrm{C}^{*}$ algebra is a simple topological algebra.

Non-abelian free groups are $\mathrm{C}^{*}$-simple. The first proof of this fact, due to Powers [Pow75], relies on a combinatorial property of free groups shared by many other groups, called for this reason Powers groups. Thus Powers groups are $\mathrm{C}^{*}$-simple. The Baumslag-Solitar group BS $(m, n)$ is $\mathrm{C}^{*}$-simple if and only if $|m| \neq|n|$ [Iva07], Theorem 4.9. In this case, it is actually a strongly Powers group [HP11], Theorem 3.iii with $|m|,|n| \geq 2$. A group $G$ is said to be strongly Powers if any of its subnormal subgroups is a Powers group. A subgroup $N$ of $G$ is a subnormal subgroup of $G$ if there are subgroups $N_{i}(1 \leq i \leq n<\infty)$ of $G$ such that $N \subset N_{1} \subset \cdots \subset N_{n-1} \subset$ $N_{n}=G$ and $N_{i}$ is normal in $N_{i+1}$ for every $i$.

Theorem 4.1. Let $|m|>1, \xi \in \mathbb{Z}_{m}$. Then $\overline{\mathrm{BS}}(m, \xi)$ is a strongly Powers group.

Amenable groups are not $C^{*}$-simple, see [Har07], Proposition 3. This is the reason why we have to exclude the groups $\overline{\mathrm{BS}}(m, \xi)$ with $|m|=1$. Actually, there is only 
one such marked group [GS08], Theorem 2.1, and it is isomorphic to the solvable group $\mathbb{Z}>\mathbb{Z}$ [Sta06a], Theorem 2 .

We consider the action of $\Gamma=\overline{\mathrm{BS}}(m, \xi)$ on its Bass-Serre tree $T=X_{m, \xi}$ and use the criterion of de la Harpe and Préaux [HP11], Corollary 15, on tree action to show Theorem 4.1. The latter criterion needs the action of $\Gamma$ to be faithful in a strong sense: it has to be slender. Two other conditions are required, namely minimality and strong hyperbolicity, but both follow immediately from [HP11], Proposition 20, as $\Gamma$ is a non-ascending HNN extension. Let us define a slender action. A tree automorphism $\gamma$ of $T$ is slender if its fixed point set $(\partial T)^{\gamma}$ has empty interior in $\partial T$ with respect to the shadow topology (Section 1.3). The action of $\Gamma$ on $T$ is slender if for every $\gamma \in \Gamma \backslash\{1\}$ the automorphism of $T$ induced by $\gamma$, also denoted by $\gamma$, is slender. A slender action is faithful, it is even strongly faithful in the sense of [HP11], Section 1.

Since hyperbolic elements are obviously slender, we focus now on the fixed point set of elliptic elements. We still need more definitions to describe the fixed point set $T^{\gamma}$ for any $\gamma \in B$.

Let $l, u \in \mathbb{Z} \cup\{ \pm \infty\}$. We denote by $\{l \leq \sigma \leq u\}$ the subgraph of $T$ whose vertices $\gamma B$ satisfy $l \leq \sigma(\gamma) \leq u$. We denote by $T[l, u]$ the connected component of $1 B$ in $\{l \leq \sigma \leq u\}$.

Lemma 4.2. Let $l, u \in \mathbb{Z} \cup\{ \pm \infty\}$. Assume either that $l>-\infty$ and $|m|>1$, or that $u<+\infty$. Then, the set $\partial\{l \leq \sigma \leq u\} \subset \partial T$ has empty interior in $\partial T$ with respect to the shadow topology.

Proof. Assume that $l>-\infty$ and $|m|>1$. Since any vertex $\gamma B$ of $T$ has $|m|$ neighbours $\gamma^{\prime} B$ such that $\sigma\left(\gamma^{\prime}\right)=\sigma(\gamma)-1$, any shadow contains (the class of) a geodesic ray $\left(\gamma_{1} B, \gamma_{2} B, \ldots, \gamma_{n} B, \ldots\right)$ such that $\sigma\left(\gamma_{n}\right)$ tends to $-\infty$. Such a ray does not lie in $\partial\{l \leq \sigma \leq u\}$. The proof of the second case is analogous (any vertex $\gamma B$ of $T$ has countably many neighbours $\gamma^{\prime} B$ such that $\left.\sigma\left(\gamma^{\prime}\right)=\sigma(\gamma)+1\right)$.

Let $v$ be the natural valuation on $\mathbb{Z}[X]$, i.e., the one defined by $v\left(X^{i}\right)=i$ for every $i$. Let $\nu$ be the "valuation" defined on $B$ by $\mu(\gamma)=\sup \left\{i \geq 1 \mid P_{\gamma}(\xi / m) \in d \hat{m}^{i} \mathbb{Z}_{m}\right\}$ where $\sup \emptyset=0$. Let $\gamma \in B$. Observe that

- $\gamma \in B_{m}$ if and only if $\mu(\gamma) \geq 1$,

- $\gamma \in B_{\xi}$ if and only if $\nu\left(P_{\gamma}\right) \geq 1$.

Proposition 4.3. Let $\gamma \in B$. We have $T^{\gamma}=T\left[-\mu(\gamma), v\left(P_{\gamma}\right)\right]$.

Corollary 4.4. Let $\Gamma=\overline{\mathrm{BS}}(m, \xi)$.

- The action of $\Gamma$ on $T$ is slender.

- The centralizer of $b$ in $\Gamma$ coincides with $B$ if $|m|>1$.

- The centralizer of $a^{k}$ in $\Gamma$ coincides with $\langle a\rangle$ for every $k \neq 0$. 
Proof. Let us show that the action of $\Gamma$ is slender. Remember that any hyperbolic element of $\Gamma$ is slender. Since any elliptic element is conjugated to some $\gamma \in B$ by a homeomorphism of $\partial T$, it suffices to prove that every $\gamma \in B \backslash\{1\}$ is slender. Since $v\left(P_{\gamma}\right)<\infty$ for every $\gamma \in B \backslash\{1\}$, the result follows from Proposition 4.3 and Lemma 4.2.

Assume that $|m|>1$ and let $\gamma$ be an element of the centralizer of $b$ in $\Gamma$. By Proposition 4.3, we have $T^{b}=1 B$. Since $\gamma$ commutes with $b$, we have then $\gamma \cdot 1 B=$ $1 B$, i.e., $\gamma \in B$.

Let $k \in \mathbb{Z} \backslash\{0\}$ and let $\gamma$ be an element of the centralizer of $a^{k}$ in $\Gamma$. Let $a_{+}$ (resp. $a_{-}$) be the class of rays which are cofinal with $\left(a^{n} B\right)_{n \geq 0}\left(\right.$ resp. $\left.\left(a^{n} B\right)_{n \leq 0}\right)$. Then $\gamma$ preserves the fixed point set $\left\{a_{-}, a_{+}\right\}$of $a^{k}$. Since $\sigma\left(\gamma a^{n}\right)=\sigma(\gamma)+n$ for every $n \in \mathbb{Z}, \gamma$ cannot exchange $a_{+}$and $a_{-}$and hence fixes them both. Consequently, there is some $n \in \mathbb{Z}$ such that $\gamma \cdot B=a^{n} B$, i.e., there is $g \in B$ such that $\gamma=a^{n} g$. We deduce that $g$ centralizes $a^{k}$ and hence $g$ fixes $a_{+}$and $a_{-}$. As $g$ is elliptic, Proposition 4.3 gives $v\left(P_{g}\right)=+\infty$, hence $g=1$. Thus $\gamma=a^{n}$.

Proposition 4.3 is a straightforward consequence of the following lemma.

Lemma 4.5. Let $g \in \Gamma$ and let $c_{0} a^{\epsilon_{1}} c_{1} \ldots a^{\epsilon_{k}} c_{k}$ with $\epsilon_{i} \in\{ \pm 1\}, c_{i} \in B$, be a reduced form of $g$. Let $\sigma_{i}(g)=\sum_{j=1}^{i} \epsilon_{j}$. Let $\gamma \in B$. The following conditions are equivalent:

(i) $g B \in T^{\gamma}$,

(ii) $g^{-1} \gamma g \in B$,

(iii) $-\mu(\gamma) \leq \sigma_{i}(g) \leq v\left(P_{\gamma}\right)$ for every $1 \leq i \leq k$.

If the previous conditions hold, then we have $g^{-1} \gamma g=a^{-\sigma(g)} \gamma a^{\sigma(g)}$.

Proof. (i) $\Longleftrightarrow$ (ii) is trivial. We show the equivalence (ii) $\Longleftrightarrow$ (iii) and the last statement of the lemma by induction on $k$. If $k=0$, both are trivial. Assume that $k>0$ and write $w=c_{0} a^{\epsilon_{1}} g^{\prime}$.

(ii) $\Longrightarrow$ (iii): We have $g^{-1} \gamma^{\prime} g^{\prime} \in B$ with $\gamma^{\prime}=a^{-\epsilon_{1}} \gamma a^{\epsilon_{1}}$. By Britton's lemma, we have $g^{\prime-1} \gamma^{\prime} g^{\prime} \in B$ and, either $\gamma \in B_{m}$ and $\epsilon_{1}=-1$, or $\gamma \in B_{\xi}$ and $\epsilon_{1}=1$. We deduce that $\gamma^{\prime} \in B, \mu\left(\gamma^{\prime}\right)=\mu(\gamma)+\epsilon_{1}, v\left(P_{\gamma^{\prime}}\right)=v\left(P_{\gamma}\right)-\epsilon_{1}, \gamma^{\prime} \in B$ and $-\mu(\gamma) \leq \epsilon_{1} \leq \nu\left(P_{\gamma}\right)$. The result then follows from the induction hypothesis.

(iii) $\Longrightarrow$ (ii): Since $-\mu(\gamma) \leq \epsilon_{1} \leq \nu\left(P_{\gamma}\right)$, we have either $\epsilon_{1}=-1$ and hence $\gamma \in B_{m}$, or $\epsilon_{1}=1$ and hence $\gamma \in B_{\xi}$. We deduce that $\gamma^{\prime} \in B$. The result follows from the induction hypothesis.

Last statement: By induction hypothesis, we have

$$
g^{\prime-1} \gamma^{\prime} g^{\prime}=a^{-\sigma\left(g^{\prime}\right)} \gamma^{\prime} a^{\sigma\left(g^{\prime}\right)}=a^{-\epsilon_{1}-\sigma\left(g^{\prime}\right)} \gamma a^{\epsilon_{1}+\sigma\left(g^{\prime}\right)}=a^{-\sigma(g)} \gamma a^{\sigma(g)} .
$$

Proof of Theorem 4.1. Since $\Gamma=\overline{\mathrm{BS}}(m, \xi)$ is a non-ascending HNN extension, the action of $\Gamma$ is strongly hyperbolic on $T$ and minimal on $\partial T$ [HP11], Proposition 20. The action of $\Gamma$ on $T$ is slender by Corollary 4.4. By [HP11], Corollary 15, $\Gamma$ is a strongly Powers group. 
Inner amenability. A countable group $G$ is said inner amenable if it admits a mean (i.e., a non-zero, finite and finitely additive measure) on the set of all the subsets of $G \backslash\{1\}$, which is invariant under inner automorphisms. We say that $G$ has the icc property if the conjugacy class of any of its non-trivial element is infinite. These two notions are motivated by the study of the von Neumann algebra $W^{*}(G)$ of $G$ (see [Eff75], [BH86]). Amenable groups or groups that do not have icc are clearly inner amenable. The second-named author has proved that the Baumslag-Solitar group BS $(m, n)$ has icc, is inner amenable but not amenable whenever $|m|>|n|>1$ [Sta06b], Examples 2.4 and 3.2. Note that for every $|m|>1$, the group $\overline{\mathrm{BS}}(m, \xi)$ also has icc since every Powers group does [Har85], Proposition 1.

Proposition 4.6. Let $|m|>1, \xi \in \mathbb{Z}_{m}$. The group $\overline{\mathrm{BS}}(m, \xi)$ is inner amenable and non-amenable.

The proof relies on:

Theorem 4.7 ([Sta06b], Proposition 0.2). Let $\Gamma=\operatorname{HNN}(\Lambda, H, K, \phi)$ with $H \neq \Lambda$ or $K \neq \Lambda$. Let $Z(\Lambda)$ be the center of $\Lambda$. If for every $n \geq 1$ there exist some nontrivial elements $h_{0}^{(n)}, h_{1}^{(n)}, \ldots, h_{n}^{(n)} \in Z(\Lambda) \cap H \cap K$ such that $h_{i}^{(n)}=\phi\left(h_{i-1}^{(n)}\right)$ for $i=1, \ldots, n$, then $\Gamma$ is inner amenable.

Proof of Proposition 4.6. Let $n \geq 1$ and set $h_{i}^{(n)}:=a^{i} b^{m^{n+1}} a^{-i}$ for $i=0, \ldots, n$. The hypotheses of Theorem 4.7. are trivially satisfied by these elements, which proves that $\overline{\mathrm{BS}}(m, \xi)$ is inner amenable. Using Britton's lemma, we can readily show that the subgroup generated by $a$ and $b a b^{-1}$ is a non-abelian free subgroup of $\overline{\mathrm{BS}}(m, \xi)$. Hence $\overline{\mathrm{BS}}(m, \xi)$ is not amenable by a classical result of von Neumann.

\section{Homomorphisms}

This section is devoted to the study of group homomorphisms from a given limit group to another one. We classify the limits of Baumslag-Solitar groups $\overline{\mathrm{BS}}(m, \xi)$ up to abstract group isomorphism (Theorem 5.11), we compute the automorphism group of every limit (Proposition 5.13) and prove that every limit is hopfian (Theorem 5.9). Finally, we show that every limit has infinite twisted conjugacy classes (Proposition 5.5)

Since the map $a \mapsto a, b \mapsto b$ induces an isomorphism from $\operatorname{BS}(m, n)$ to $\mathrm{BS}(-m,-n)$, it also induces an isomorphism from $\overline{\mathrm{BS}}(m, \xi)$ to $\overline{\mathrm{BS}}(-m,-\xi)$ for any $\xi \in \mathbb{Z}_{m}$. For this reason, we will assume that $m>0$.

The following lemma can be readily deduced from the definition of $\overline{\mathrm{BS}}(m, \xi)$.

Lemma 5.1. Let $d=\operatorname{gcd}(m, \xi)$ and let $\eta=\pi(\xi / d)$ where $\pi: \mathbb{Z}_{m} \rightarrow \mathbb{Z}_{\hat{m}}$ is the canonical map. The map $a \mapsto a, b \mapsto b^{d}$ induces an injective homomorphism from $\overline{\mathrm{BS}}(\hat{m}, \eta)$ into $\overline{\mathrm{BS}}(m, \xi)$. 
From now on we will consider $\widetilde{\mathrm{BS}}(m, \xi)$ (see Introduction or paragraph before Corollary 3.2) rather than $\overline{\mathrm{BS}}(m, \xi)$ because it will ease off notations. We fix $m, m^{\prime} \in$ $\mathbb{N} \backslash\{0\}$ and $\xi \in \mathbb{Z}_{m}, \xi^{\prime} \in \mathbb{Z}_{m^{\prime}}$ and we set $\Gamma=\widetilde{\mathrm{BS}}(m, \xi)$ and $\Gamma^{\prime}=\widetilde{\mathrm{BS}}\left(m^{\prime}, \xi^{\prime}\right)$. Recall that $\sigma: \Gamma \rightarrow \mathbb{Z}$ is the homomorphism defined by $\sigma(a)=1$ and $\sigma(b)=0$. We denote also by $\sigma$ the analogous homomorphism from $\Gamma^{\prime}$ onto $\mathbb{Z}$.

The following proposition shows that every surjective homomorphism from $\Gamma$ onto $\Gamma^{\prime}$ is conjugated to a simple one by an element of $\Gamma^{\prime}$.

Proposition 5.2. Let $p: \Gamma \rightarrow \Gamma^{\prime}$ be a surjective homomorphism. Then $m^{\prime}$ divides $m$, $\sigma(p(a))= \pm 1$ and $p\left(e_{0}\right)$ is conjugated to $\pm e_{0}$. Moreover $\sigma(p(a))=1$ if $m^{\prime}>1$.

Before proving Proposition 5.2, we quote the following observation for further reference.

Remark 5.3. For any $Q \in \mathbb{Z}\left[X^{ \pm 1}\right]$, there exists $\psi_{Q} \in \operatorname{Aut}\left(\mathbb{Z}\left[X^{ \pm 1}\right] \rtimes \mathbb{Z}\right)$ such that $\psi_{Q}(1,0)=(1,0)$ and $\psi_{Q}(0,1)=(Q, 1)$. This automorphism satisfies $\psi_{Q}(P, 0)=$ $(P, 0)$ for all $P \in \mathbb{Z}\left[X^{ \pm 1}\right]$.

Indeed, this can be readily checked with the well-known presentation

$$
\left.\mathbb{Z}\left[X^{ \pm 1}\right] \rtimes \mathbb{Z}=\mathbb{Z} \imath \mathbb{Z}=\langle a, b|\left[a^{i} b a^{-i}, b\right]=1 \text { for all } i \geq 1\right\rangle,
$$

where $a$ corresponds to $(0,1)$ and $b$ corresponds to $(1,0)$.

Proof of Proposition 5.2. We set $\alpha=p(a), \beta=p\left(e_{0}\right)$ and $\gamma=\alpha \beta \alpha^{-1}$. First we show that $\beta$ is an elliptic element of $\Gamma^{\prime}$. Assume by contradiction that $\beta$ is hyperbolic. Then $\gamma^{m}$ is hyperbolic with axis $\alpha(D)$, where $D$ is the axis of $\beta$. Since $e_{1}=a\left(m e_{0}\right) a^{-1}$ commutes with $e_{0}$ in $\Gamma, \beta$ commutes with $\gamma^{m}$. As a result, $\gamma^{m}$ has the same axis as $\beta$, namely $D$. Thus $D$ is invariant under $\alpha$. There are two cases: either $\alpha$ is hyperbolic (case 1 ) or $\alpha$ is elliptic (case 2).

Case 1: the bi-infinite ray $D$ is then the axis of $\alpha$. As $\alpha$ and $\beta$ generate $\Gamma^{\prime}$, the two ends of $D$ are fixed ends of $\Gamma^{\prime}$. This is impossible since a non-degenerate HNN extension has at most one fixed end on the boundary of its Bass-Serre tree.

Case 2: the automorphism $\alpha^{\prime}=\alpha \beta$ is hyperbolic, since $\Gamma^{\prime}$ cannot be generated by two elliptic elements. Indeed, the set of elliptic elements is contained in the kernel of $\sigma: \Gamma^{\prime} \rightarrow \mathbb{Z}$. So the argument used in case 1 applies to $\alpha^{\prime}$ and $\beta$.

Therefore, up to conjugacy, we can assume that $\beta \in E$. Now we use the surjective homomorphism $q_{m^{\prime}, \xi^{\prime}}: \Gamma^{\prime} \rightarrow \mathbb{Z} \prec \mathbb{Z}$ (see Proposition 2.9(v)). Since $\sigma(\beta)=0$ and $\{\sigma(\alpha), \sigma(\beta)\}$ generates $\mathbb{Z}$, we have $\sigma(\alpha)=\epsilon^{\prime}$ with $\epsilon^{\prime}= \pm 1$. We can write $q(\beta)=(P, 0), q(\alpha)=\left(Q, \epsilon^{\prime}\right)$ with $P, Q \in \mathbb{Z}\left[X^{ \pm 1}\right]$. If $\epsilon^{\prime}=1$, the automorphism $\psi_{-Q}$, defined as in Remark 5.3, maps $(P, 0)$ to $(P, 0)$ and $(Q, 1)$ to $(0,1)$; if $\epsilon^{\prime}=1$, the automorphism $\psi_{X Q}$ maps $(P, 0)$ to $(P, 0)$ and $(Q,-1)$ to $(0,-1)$. In both cases, the image $\left\{(P, 0),\left(0, \epsilon^{\prime}\right)\right\}$ of $\{q(\alpha), q(\beta)\}$ by the latter automorphism generates $\mathbb{Z} \imath \mathbb{Z}$, i.e., the subgroup $P \mathbb{Z}\left[X^{ \pm 1}\right] \rtimes \mathbb{Z}$ coincides with $\mathbb{Z} \imath \mathbb{Z}$. This implies that $P$ is invertible 
in $\mathbb{Z}\left[X^{ \pm 1}\right]$, which gives $P=\epsilon X^{i}$ for some $\epsilon= \pm 1, i \in \mathbb{Z}$. As $P=q(\beta)$ lies in $\mathbb{Z}[X]$, we have $i \in \mathbb{N}$, whence $q(\beta)=q\left(a^{i}\left(\epsilon e_{0}\right) a^{-i}\right)$. Since $q$ is injective on $E$ by Proposition 2.9, we deduce that $\beta=a^{i}\left(\epsilon e_{0}\right) a^{-i}$. Thus, up to conjugacy, we can assume that $\beta=\epsilon e_{0}$.

Assume now that $m^{\prime}>1$. Let $\alpha=c_{1} a^{\epsilon_{1}} c_{2} a^{\epsilon_{2}} \ldots c_{l} a^{\epsilon_{l}} c_{l+1}=z a^{\epsilon_{l}} c_{l+1}$ (with $\epsilon_{i}= \pm 1$ and $\left.c_{i} \in E\right)$ be a reduced form in $\Gamma^{\prime}$. As $\gamma^{m}=\alpha\left(m \epsilon e_{0}\right) \alpha^{-1}$ commutes with $\beta=\epsilon e_{0}$, we deduce from Corollary 4.4 that $\alpha\left(m e_{0}\right) \alpha^{-1} \in E$. It follows that $\epsilon_{l}=1$ and $m^{\prime}$ divides $m$ by Britton's lemma. As $a^{\sigma(\alpha)}\left(m e_{0}\right) a^{-\sigma(\alpha)} \in E$ by Lemma 4.5, it follows from Britton's lemma that $\sigma(\alpha) \geq 0$. Since $\sigma(\alpha)=\sigma(z)+1 \in\{ \pm 1\}$, we deduce that $\sigma(z)=0$, which completes the proof.

Remark 5.4. For $p$ as in Proposition 5.2, note that we can write $p(a)=z a e$ with $e \in E$ and $z \in \Gamma^{\prime}$ such that $\sigma(z)=0$ when $m^{\prime}>1$; see the proof above.

Let $G$ be a group and let $\phi$ be an automorphism of $G$. Two elements $\gamma, \gamma^{\prime} \in G$ are said to be $\phi$-twisted conjugate if there is $g \in G$ such that $\gamma^{\prime}=g \gamma \phi\left(g^{-1}\right)$. We say that $G$ has infinitely many twisted conjugacy classes if $G$ has infinitely many $\phi$-twisted conjugacy classes for every automorphism $\phi$. The study of this property is mainly motivated by topological fixed point theory and by the problem of finding a twisted analogue of the classical Burnside-Frobenius theorem (see [FH94] for a introduction to these topics). Baumslag-Solitar groups $\mathrm{BS}(m, n)$ with $(m, n) \neq \pm(1,1)$ [FG06], [FG08] and the wreath product $\mathbb{Z} \imath \mathbb{Z}$ [GW06], Corollary 4.3, have infinitely many twisted conjugacy classes (the reader may consult [Rom] for an up-to-date list of known examples).

Corollary 5.5. The group $\Gamma$ has infinitely many twisted conjugacy classes.

Proof. Let $\phi$ be an automorphism of $\Gamma$. It follows from Proposition 5.2 that $\sigma \circ \phi=\sigma$. Thus $\sigma$ is constant on each $\phi$-twisted conjugacy class. As $\sigma$ takes infinitely many values, $\Gamma$ has infinitely many $\phi$-twisted conjugacy classes.

For every $i \geq 1$, we set

$$
w_{i}=a^{i}\left(m e_{0}\right) a^{-1}\left(-r_{1}(\xi) e_{0}\right) a^{-1}\left(-r_{2}(\xi) e_{0}\right) a^{-1}\left(-r_{i-1}(\xi) e_{0}\right) a^{-1} .
$$

Then $e_{i}=w_{i}$ holds in $\Gamma$ for every $i \geq 1$. Recall that

$$
\begin{aligned}
\Gamma= & \left\langle a, e_{0}, e_{1}, \ldots\right|\left[e_{i}, e_{j}\right]=1 \text { for all } i, j \geq 0, \\
& \left.a\left(m e_{0}\right) a^{-1}=e_{1}, a\left(e_{i}-r_{i}(\xi) e_{0}\right) a^{-1}=e_{i+1} \text { for all } i \geq 1\right\rangle \\
= & \left.\left\langle a, e_{0}\right|\left[e_{0}, w_{i}\right]=1 \text { for all } i \geq 1\right\rangle
\end{aligned}
$$

by Corollary 2.10 and Theorem 3.1.

We now turn to the classification of limits up to group isomorphism. To carry it out, we still need two lemmas. Let $J$ be the map defined by $J(a)=a$ and $J\left(e_{0}\right)=-e_{0}$. It follows from presentation (7) that $J$ induces an automorphism of $\Gamma$ such that $J\left(e_{i}\right)=-e_{i}$ for every $i$. 
Lemma 5.6. Let $t_{1}, \ldots, t_{n} \in\{0, \ldots, m-1\}$ and let

$$
w\left(m, t_{1}, \ldots, t_{n}\right)=a^{n+1}\left(m e_{0}\right) a^{-1}\left(-t_{1} e_{0}\right) a^{-1}\left(-t_{2} e_{0}\right) a^{-1} \ldots\left(-t_{n} e_{0}\right) a^{-1} .
$$

Then the following statements are equivalent:

(i) The image of $w\left(m, t_{1}, \ldots, t_{n}\right)$ in $\Gamma$ belongs to $E$.

(ii) The image of $w\left(m, t_{1}, \ldots, t_{n}\right) e_{0} w\left(-m,-t_{1}, \ldots,-t_{n}\right)\left(-e_{0}\right)$ in $\Gamma$ is trivial.

(iii) $t_{i}=r_{i}(\xi)$ for every $1 \leq i \leq n$.

Proof. (i) $\Longrightarrow$ (ii): One has $w\left(m, t_{1}, \ldots, t_{n}\right) e_{0} w\left(-m,-t_{1}, \ldots,-t_{n}\right)\left(-e_{0}\right)=1$ in $\Gamma$ since $J\left(w\left(m, t_{1}, \ldots, t_{n}\right)\right)=w\left(-m,-t_{1}, \ldots,-t_{n}\right)$ and $J$ maps any element of $E$ to its inverse.

(ii) $\Longrightarrow$ (iii): Assume that $w=w\left(m, t_{1}, \ldots, t_{n}\right) e_{0} w\left(-m,-t_{1}, \ldots,-t_{n}\right)\left(-e_{0}\right)$ has a trivial image in $\Gamma$. We prove the following claim by induction: we have $w=$ $v_{i} e_{0} J\left(v_{i}\right)\left(-e_{0}\right)$, with $v_{i}=a^{n+1-i}\left(e_{i}-t_{i} e_{0}\right) a^{-1}\left(-t_{i+1} e_{0}\right) a^{-1} \ldots\left(-t_{n} e_{0}\right) a^{-1}$, for every $1 \leq i \leq n$, and $t_{j}=r_{j}(\xi)$ for every $1 \leq j \leq i$. For $i=1$, the claim follows from the relation $a\left(m e_{0}\right) a^{-1}=e_{1}$ in $\Gamma$. Assume that the claim holds for some $i \geq 1$. We then have $w=v_{i} e_{0} J\left(v_{i}\right)\left(-e_{0}\right)=1$ in $\Gamma$. By Britton's lemma, the sequence $\left(a, e_{i}-t_{i} e_{0}, a^{-1}\right)$ is not reduced and hence $e_{i}-t_{i} e_{0} \in E_{m, \xi}$. Therefore $t_{i}=r_{i}(\xi)$ and $a\left(e_{i}-t_{i} e_{0}\right) a^{-1}=e_{i+1}$. We deduce that $w=v_{i+1} e_{0} J\left(v_{i+1}\right)$ with $v_{i+1}=a^{n-i}\left(e_{i+1}-t_{i+1} e_{0}\right) a^{-1}\left(-t_{i+2} e_{0}\right) \ldots\left(-t_{n} e_{0}\right) a^{-1}$, which completes the induction.

(iii) $\Longrightarrow$ (i): this follows from the fact that $w\left(m, r_{1}(\xi), \ldots, r_{i}(\xi)\right)=w_{i}$ and $w_{i}=e_{i}$ in $\Gamma$ for $i \geq 1$.

Lemma 5.7. Assume that $m=m^{\prime}>1$. Let $e \in E, z \in \Gamma^{\prime}$ such that $\sigma(z)=0$ and let $\theta$ be the map defined by $\theta(a)=z a e, \theta\left(e_{0}\right)=e_{0}$. If $\theta$ induces an homomorphism from $\Gamma$ to $\Gamma^{\prime}$ then $r_{i}(\xi)=r_{i}\left(\xi^{\prime}\right)$ for every $i \geq 1$. In this case, the restriction of $\theta$ to $E$ is the identity and we have $|\theta(\gamma)|_{a}=|\gamma|_{a}|\theta(a)|_{a}$ for every $\gamma \in \Gamma$. In particular $\theta$ is injective.

Proof. Assume that $\theta$ induces an homomorphism from $\Gamma$ to $\Gamma^{\prime}$. We show by induction that $\theta\left(e_{i}\right)=w_{i}$ for every $i \geq 1$. First observe that $\theta\left(e_{i}\right)$ commutes with $\theta\left(e_{0}\right)=$ $e_{0}$ for every $i \geq 1$. Hence $\theta\left(e_{i}\right) \in E$ for every $i \geq 1$ by Corollary 4.4. In particular, $\theta\left(e_{1}\right)=\theta\left(w_{1}\right)=z a\left(m e_{0}\right) a^{-1} z^{-1} \in E$. As $\sigma(z)=0$, we deduce from Lemma 4.5 that $\theta\left(e_{1}\right)=w_{1}$. Assume now that $\theta\left(e_{i}\right)=w_{i}$ for some $i \geq 1$. As $e_{i+1}=a\left(e_{i}-r_{i}(\xi) e_{0}\right) a^{-1}$ in $\Gamma$, we have $\theta\left(e_{i+1}\right)=z a\left(w_{i}-r_{i}(\xi) e_{0}\right) a^{-1} z^{-1} \in E$. We obtain $\theta\left(e_{i+1}\right)=w_{i+1}$ by Lemma 4.5 , which completes the induction. As $w_{i}=\theta\left(e_{i}\right) \in E$ for every $i \geq 1$, we deduce from Lemma 5.6 that $r_{i}(\xi)=r_{i}\left(\xi^{\prime}\right)$ for every $i \geq 1$.

Assume that the latter condition holds and let $\theta(a)=c_{1} a^{\epsilon_{1}} c_{2} a^{\epsilon_{2}} \ldots c_{l} a^{\epsilon_{l}} c_{l+1}$ (with $\epsilon_{j}= \pm 1$ and $c_{j} \in E$ for every $j$ ) be a reduced form in $\Gamma^{\prime}$. Because $\theta(a)\left(m e_{0}\right) \theta(a)^{-1}=e_{1}$ and $\theta(a)^{-1} e_{1} \theta(a)=m e_{0}$, we have $\epsilon_{1}=\epsilon_{l}=1$ by Britton's lemma. It readily follows that $|\theta(\gamma)|_{a}=|\gamma|_{a}|\theta(a)|_{a}$ for every $\gamma \in \Gamma$. 
Hopf property and residual finiteness. An important reason for considering Baumslag-Solitar groups was that originally they were the first examples of nonhopfian one-relator groups [BS62]. Let us recall that a group $G$ is hopfian if every surjective endomorphism from $G$ is an isomorphism. It is known that the Hopf property is neither open [ $\left.\mathrm{ABL}^{+} 05\right]$, [Sta06a] nor closed [CGP07], Proposition 5.10, in the space of marked groups.

A group $G$ is said to be residually finite if for every non-trivial element $g$ of $G$ there is a finite quotient $F$ of $G$ such that the image of $g$ in $F$ is non-trivial. For $m=1$, the only limit is $\widetilde{\mathrm{BS}}(1,0)=\mathbb{Z} \imath \mathbb{Z}$. This group is residually finite, hence hopfian by a well-known theorem of Malcev; see e.g. [LS77], Theorem IV.4.10.

Definition 5.8. A group $G$ is co-hopfian if every injective homomorphism from $G$ to itself is an isomorphism.

Theorem 5.9. The group $\Gamma$ is hopfian but not co-hopfian.

We denote by $J$ the automorphism of $\Gamma$ defined by $J(a)=a$ and $J\left(e_{0}\right)=-e_{0}$.

Proof. We can assume that $m>1$. Let $p$ be a surjective endomorphism of $\Gamma$. By Proposition 5.2, there is $\epsilon \in\{0,1\}$ and an inner automorphism $\tau$ of $\Gamma$ such that $p^{\prime}=J^{\epsilon} \circ \tau \circ p$ satisfies $p^{\prime}(a)=z a e, p^{\prime}\left(e_{0}\right)=e_{0}$ with $e \in E, z \in \Gamma$ such that $\sigma(z)=0$. By Lemma 5.7, $p^{\prime}$ is injective and hence so is $p$. Therefore $p$ is an isomorphism.

Let $k \in \mathbb{N} \backslash\{1\}$ be coprime with $m$ and let $\theta$ be the map defined by $\theta(a)=a$, $\theta\left(e_{0}\right)=k e_{0}$. Considering the group presentation (7), we deduce that $\theta$ induces an endomorphism of $\Gamma$. We can readily check that $\theta(e)=k e$ for every $e \in E$ and that $|\theta(\gamma)|_{a}=|\gamma|_{a}$ for every $\gamma \in \Gamma$. It follows that $\theta$ is injective. We also deduce that $\theta(\Gamma) \cap E=\theta(E)=k E \neq E$. Hence $\theta$ is not surjective.

We say that $\xi \in \mathbb{Z}_{m}=\bigoplus_{p \mid m} \mathbb{Z}_{p}$ is algebraic if the $p$-component $\xi_{p} \in \mathbb{Z}_{p}$ of $\xi$ is algebraic over $\mathbb{Q}$ for every prime $p$ dividing $m$. It only means that $\xi$ is a root in $\mathbb{Z}_{m}$ of some polynomial with coefficients in $\mathbb{Z}$. Let $d=\operatorname{gcd}(m, \xi)$ and $\eta=\pi(\xi / d)$ where $\pi: \mathbb{Z}_{m} \rightarrow \mathbb{Z}_{\hat{m}}$ is the canonical map. It readily follows from the definition of $\chi$ (resp. $\hat{\chi}$ ) in Proposition 2.9 (vi) that $\xi$ (resp. $\eta$ ) is algebraic if and only if ker $\chi \neq 1$ (resp. $\operatorname{ker} \hat{\chi} \neq 1$ ). If $\xi$ is invertible in $\mathbb{Z}_{m}$, i.e., $\operatorname{gcd}(m, \xi)=1$, then the two kernels coincide with $\bigcap_{i \geq 0} a^{-i} E a^{i}$.

Proposition 5.10. If $\eta$ is algebraic, then $\Gamma$ is not residually finite.

Proof. By Lemma 5.1, $\overline{\mathrm{BS}}(m, \xi)(\hat{m}, \eta)$ embeds isomorphically into $\overline{\mathrm{BS}}(m, \xi)$. Since a subgroup of a residually finite group is residually finite, we can assume that $\operatorname{gcd}(m, \xi)=1$. By Proposition 2.9(vi), we can pick $e \in \bigcap_{i \geq 0} a^{-i} E a^{i} \backslash E_{1}$. We set $\gamma=\left[e, a e_{0} a^{-1}\right]=(-e) a\left(-e_{0}\right) a^{-1} e a e_{0} a^{-1}$. By Britton's lemma, $\gamma$ is not trivial in $\Gamma$. We show that $\gamma$ has trivial image in any finite quotient of $\Gamma$, which 
proves that $\Gamma$ is not residually finite. Let $F$ be a finite quotient of $\Gamma$ with cardinal $n$. There is $e^{\prime} \in E$ such that $e=a^{-n+1} e^{\prime} a^{n-1}$ in $\Gamma$. Since $a^{n}=1$ in $F$, we have $\gamma=\left[a^{-n+1} e^{\prime} a^{n-1}, a e_{0} a^{-1}\right]=a\left[e^{\prime}, e_{0}\right] a^{-1}=1$ in $F$. The proof is then complete.

Classification of limits up to group isomorphism. First recall that we assume $m, m^{\prime}>0$.

Theorem 5.11. The group $\Gamma$ is isomorphic to $\Gamma^{\prime}$ if and only if $m=m^{\prime}$ and $r_{i}(\xi)=$ $r_{i}\left(\xi^{\prime}\right)$ for every $i \geq 1$.

Proof. Assume that $\Gamma$ is isomorphic to $\Gamma^{\prime}$. If $m^{\prime}=1$, then $\Gamma^{\prime}$ is isomorphic to $\mathbb{Z} \geq \mathbb{Z}$ and so is $\Gamma$. This forces $m=1$ for otherwise $\Gamma$ would contain a non-abelian free subgroup. It follows that $r_{i}(\xi)=r_{i}\left(\xi^{\prime}\right)=0$ for every $i \geq 1$. Therefore we can assume that $m^{\prime}>1$. By Proposition 5.2, $m^{\prime}$ divides $m$, and there is an isomorphism $\theta: \Gamma \rightarrow \Gamma^{\prime}$ such that $\theta(a)=z a e, \theta\left(e_{0}\right)=e_{0}$ with $e \in E, z \in \Gamma$ such that $\sigma(z)=0$. Considering $\theta^{-1}$, we also deduce that $m$ divides $m^{\prime}$ and hence $m=m^{\prime}$. By Lemma 5.7, we have $r_{i}(\xi)=r_{i}\left(\xi^{\prime}\right)$ for every $i \geq 1$.

The converse follows immediately from the group presentation (7).

Automorphism group. Let $e \in E$ and let $\phi_{e}$ be the map $\Gamma$ defined by $\phi_{e}(a)=a e$ and $\phi\left(e_{0}\right)=e_{0}$. We deduce from the group presentation (8) that $\phi_{e}$ induces an automorphism of $\Gamma$ with inverse map $\phi_{-e}$. Moreover, we have $J \circ \phi_{e} \circ J=\phi_{-e}$. The following lemma is then immediate.

Lemma 5.12. The automorphisms $\phi_{e_{0}}$ and $J$ generate a group isomorphic to an infinite dihedral group, namely the semi-direct product $\mathbb{Z} e_{0} \rtimes \mathbb{Z} / 2 \mathbb{Z}$, where the action of $\mathbb{Z} / 2 \mathbb{Z}$ on $E$ is multiplication by -1 .

Hence we can consider the semi-direct product $\Gamma \rtimes\left(\mathbb{Z} e_{0} \rtimes \mathbb{Z} / 2 \mathbb{Z}\right)$ where the action of $\mathbb{Z} e_{0} \rtimes \mathbb{Z} / 2 \mathbb{Z}$ on $\Gamma$ is the obvious one. We denote by $\operatorname{Inn}(\Gamma)$ the group of inner automorphisms of $\Gamma$ and by $\operatorname{Out}(\Gamma)=\operatorname{Aut}(\Gamma) / \operatorname{Inn}(\Gamma)$ the group of outer automorphisms.

Proposition 5.13. Assume that $m>1$.

- $\operatorname{Out}(\Gamma)$ is isomorphic to $\mathbb{Z} e_{0} \rtimes \mathbb{Z} / 2 \mathbb{Z}$.

- $\operatorname{Aut}(\Gamma)$ is isomorphic to $\Gamma \rtimes\left(\mathbb{Z} e_{0} \rtimes \mathbb{Z} / 2 \mathbb{Z}\right)$.

We denote by $C$ the subgroup of $E$ generated by $e_{1}-m e_{0}$ and the elements $e_{i}-e_{i-1}+r_{i-1} e_{0}$ with $i \geq 1$. Proposition 5.13 will follow from:

Lemma 5.14. Assume that $m>1$ and let $e \in E$.

- The image of $\phi_{e} \circ J$ in $\operatorname{Out}(\Gamma)$ is non-trivial. 
- The image of $\phi_{e}$ is trivial in $\operatorname{Out}(\Gamma)$ if and only if $e \in C$.

- For every automorphism $\phi$ of $\Gamma$, there is $e \in E, \epsilon \in\{0,1\}$ such that $\phi=\phi_{e} \circ J^{\epsilon}$ holds in $\operatorname{Out}(\Gamma)$.

Proof. Since $\Gamma$ is centerless (see e.g. Corollary 4.4) and torsion-free, $\operatorname{Inn}(\Gamma)$ is torsion-free. The first assertion follows from the fact that $\phi_{e} \circ J$ has order 2 .

Let $e \in E$ and assume that there is $z \in \Gamma$ such that $\phi_{e}=\tau_{z}$. As $z$ centralizes $e_{0}$, we deduce from Corollary 4.4 that $z \in E$. We deduce from the equality $\phi_{e}(a)=$ $z a z^{-1}$ that $a(e+z) a^{-1}=z$. By Britton's lemma, we have $e+z \in E_{m, \xi}$ and hence $z \in E_{1}$. Identifying $E$ with $B$, we deduce from Proposition 2.9.v, that $X\left(P_{e}(X)+\right.$ $\left.P_{z}(X)\right)=P_{z}(X)$. Therefore $P_{e}(X)=-\frac{X-1}{X} P_{z}(X)=\iota\left(-P_{z}(X)\right) \in \mathfrak{C}$ where $\iota$ and $\mathfrak{C}$ are defined in Proposition 2.9 (vii). Since $\mathfrak{C}=q(C)$ and $q$ in injective, we have $e \in C$. Conversely, if $e \in C$, we can readily check that $\phi_{e}=\tau_{z}$ where $z \in E_{1}$ is given by the formula $P_{z}(X)=-\frac{X}{X-1} P_{e}(X)$.

Consider now an arbitrary automorphism $\phi$ and let us show that $\phi=\phi_{e} \circ J^{\epsilon}$ holds in $\operatorname{Out}(\Gamma)$ for some $e \in E$ and some $\epsilon \in\{0,1\}$. By Proposition 5.2, we can assume that $\phi\left(e_{0}\right)= \pm e_{0}$ and $\phi(a)=z a e^{\prime}$ with $e^{\prime} \in E$ and $z$ such that $\sigma(z)=0$. Composing possibly by $J$, we can assume that $\phi\left(e_{0}\right)=e_{0}$ hence that $\phi$ and $\phi^{-1}$ both satisfy the conditions of Lemma 5.7. We deduce from Lemma 5.7 that $1=|a|_{a}=|\phi(a)|_{a}\left|\phi^{-1}(a)\right|_{a}$. Therefore $|z|_{a}=0$, i.e., $z \in E$ and hence $\phi=\phi_{e^{\prime}+z}$ holds in $\operatorname{Out}(\Gamma)$.

Proof of Proposition 5.13. By Lemma 5.14, Out $(\Gamma)$ is generated by the images of $J, \phi_{e}$ with $e \in E$. By Proposition 2.9(vii), the quotient $E / C$ is infinite cyclic and generated by the image of $e_{0}$. Hence, by Lemma 5.14, the natural map $\operatorname{Aut}(\Gamma) \rightarrow$ $\operatorname{Out}(\Gamma)$ induces an isomorphism from the subgroup generated by $J$ and $\phi_{e_{0}}$ onto $\operatorname{Out}(\Gamma)$. It follows then from Lemma 5.12 that $\operatorname{Out}(\Gamma)$ is isomorphic to $\mathbb{Z} e_{0} \rtimes \mathbb{Z} / 2 \mathbb{Z}$. As a result, the exact sequence $1 \rightarrow \operatorname{Inn}(\Gamma) \rightarrow \operatorname{Aut}(\Gamma) \rightarrow \operatorname{Out}(\Gamma) \rightarrow 1$ splits. Since $\Gamma$ is centerless, it naturally identifies with $\operatorname{Inn}(\Gamma)$. Thus $\operatorname{Aut}(\Gamma)$ is isomorphic to $\Gamma \rtimes\left(\mathbb{Z} e_{0} \rtimes \mathbb{Z} / 2 \mathbb{Z}\right)$.

An immediate consequence of Proposition 5.13 is that every automorphism of $\Gamma$ is induced by an automorphism of $\mathbb{F}\left(a, e_{0}\right)$. Group presentations with such property are called almost quasi-free presentations [LS77], Chapter II.2.

Recall that the map $a \mapsto(0,1), e_{0} \mapsto(1,0)$ induces a surjective homomorphism $q_{m, \xi}$ from $\Gamma$ onto $\mathbb{Z} \imath \mathbb{Z}=\widetilde{\mathrm{BS}}(1,0)$. Another consequence of Proposition 5.13 is:

Corollary 5.15. If $|m|>1$, then the kernel of $q_{m, \xi}$ is a characteristic free subgroup of $\Gamma$ of infinite rank.

Proof. The normal subgroup $N=\operatorname{ker} q_{m, \xi}$ is a free group by [GS08], Theorem 3.11. Because $N \nsubseteq E_{m, \xi}$ and $N E_{m, \xi}$ has infinite index in $\Gamma, N$ is not finitely generated by [KS71], Theorem 9 . 
To conclude, due to Proposition 5.13, it suffices to show that $N$ is invariant under the automorphisms $J$ and $\phi_{e_{0}}$. It is invariant under $J$ since the diagram

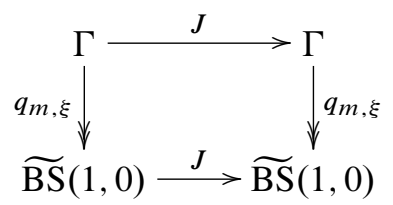

commutes. A similar argument works for the automorphism $\phi_{e_{0}}$.

Equationally noetherian groups. In this section we determine which groups $\overline{\mathrm{BS}}(m, \xi)$ are equationally noetherian. Equationally noetherian groups play an important role in algebraic geometry over groups [BMR99], the state-of-the-art approach to equations over groups. An equationally noetherian group enjoys the following strong form of the Hopf property: any sequence of surjective endomorphisms is stationary (see [MR00], Theorem D1.2, or [OH07], Corollary 2.8). Let us recall the definition. Given $w=w\left(x_{1}, \ldots, x_{n}\right) \in G * \mathbb{F}\left(x_{1}, \ldots, x_{n}\right)$ and an $n$-tuple $\left(g_{1}, \ldots g_{n}\right) \in G^{n}$, we denote by $w\left(g_{1}, \ldots, g_{n}\right)$ the element of $G$ obtained by replacing $x_{i}$ by $g_{i}$. For any subset $W \subseteq G * \mathbb{F}\left(x_{1}, \ldots, x_{n}\right)$, we consider the roots

$$
\operatorname{Root}(W)=\left\{\left(g_{1}, \ldots, g_{n}\right) \in G^{n} \mid w\left(g_{1}, \ldots, g_{n}\right)=1 \text { for all } w \in W\right\} .
$$

Definition 5.16. A group $G$ is equationally noetherian if, for all $n \geqslant 1$ and for all $W \subseteq G * \mathbb{F}\left(x_{1}, \ldots, x_{n}\right)$, there exists a finite subset $W_{0} \subseteq W$ such that $\operatorname{Root}(W)=$ $\operatorname{Root}\left(W_{0}\right)$.

Linear groups over a commutative, noetherian, unitary ring (e.g. a field), are equationally noetherian [Bry77], [Gub86] while any wreath product of a non-abelian group by an infinite one is not equationally noetherian [BMR97].

Proposition 5.17. Let $m \in \mathbb{Z} \backslash\{0\}$ and $\xi \in \mathbb{Z}_{m}$. The group $\overline{\mathrm{BS}}(m, \xi)$ is equationally noetherian if and only if $|m|=1$.

We need the following result on Baumslag-Solitar groups.

Proposition 5.18 ([BMR99], p. 41, Proposition 5). Let $m, n \in \mathbb{Z} \backslash\{0\}$.

(1) If either $|m|=1$ or $|n|=1$ or $|m|=|n|$, then the group $\operatorname{BS}(m, n)$ is linear over $\mathbb{R}$ and hence equationally noetherian.

(2) In all other cases the group $\mathrm{BS}(m, n)$ is not equationally noetherian.

Since we need some excerpts of the proof of Proposition 5.18, we provide it in full. Let $R$ be a commutative ring with unity. We will use the following fact without further mention. If a group $G$ has a finite index subgroup which is linear over $R$, then so is $G$; see [Weh73], Lemma 2.3. 
Proof of Proposition 5.18. (1) Suppose first that $|m|=1$ or $|n|=1$. It is well known, and easy to show, that the map $a \mapsto\left(x \mapsto \frac{m}{n} x\right), b \mapsto(x \mapsto x+1)$ yields an injective group homomorphism from $\operatorname{BS}(m, n)$ into the affine group over $\mathbb{R}$. The group $\mathrm{BS}(m, n)$ is then linear over $\mathbb{R}$. As $\mathrm{BS}(m, n)$ is soluble in this case, we observe that it is linear over $\mathbb{Z}$ if and only if it is polycyclic ${ }^{2}$, i.e., $|n|=|m|=1$.

If $m=n$, it is easy to check that the normal subgroup $\left\langle\left\langle a, b^{m}\right\rangle\right\rangle$ is isomorphic to $\mathbb{F}_{|m|} \times \mathbb{Z}$ and hence linear over $\mathbb{Z}$. Clearly, it has index $|m|$ in $\operatorname{BS}(m, m)$. Thus $\operatorname{BS}(m, n)$ is linear over $\mathbb{Z}$.

Suppose finally that $n=-m$. Let $B S_{2}(m, n)=\left\langle\left\langle a^{2}, b\right\rangle\right\rangle \subset \operatorname{BS}(m, n)$. The subgroups $B_{2}(m, m)$ and $B_{2}(m,-m)$ are clearly isomorphic and have index two in $\mathrm{BS}(m, m)$ and $\mathrm{BS}(m,-m)$, respectively. We have shown that $\mathrm{BS}(m, m)$ is linear over $\mathbb{Z}$. We deduce that $B_{2}(m, m)$ is linear over $\mathbb{Z}$ and hence so is $\operatorname{BS}(m,-m)$.

It follows from the above proof that $\operatorname{BS}(m, n)$ is linear over $\mathbb{Z}$ if and only if $|m|=|n|$.

(2) Since the groups $\mathrm{BS}(m, n)$ and $\mathrm{BS}(n, m)$ are isomorphic, we may assume that $|m|<|n|$. Then there exists $v>0$ and a prime number $p$ such that $p^{v}$ divides $n$ but not $m$. Let us consider the set

$$
W=\left\{w_{i}:=\left[x^{-i} y x^{i}, z\right] \mid i \in \mathbb{N} \backslash\{0\}\right\} \subseteq \mathbb{F}(x, y, z)
$$

and the triples $\left(x_{k}=a, y_{k}=b^{n^{k}}, z_{k}=b\right){ }^{3}$ If $n$ divides an integer $\beta$, then we have $a^{-1} b^{\beta} a=b^{\beta^{\prime}}$ and the factorization of $\beta^{\prime}$ contains (strictly) less factors $p$ than that of $\beta$. Consequently, for all $k$, there exists $N(k) \in \mathbb{N}$ and $\alpha(k) \in \mathbb{Z}$ such that

$$
a^{-N(k)} b^{n^{k}} a^{N(k)}=b^{\alpha(k)} \text { and } n \text { does not divide } \alpha(k) .
$$

Remark 5.19. 1 . Since $a^{-k} b^{n^{k}} a^{k}=b^{m^{k}}$, we have $N(k) \geqslant k$.

2. Set $\mu=\mu(m)$ to be the maximal exponent arising in the factorization of $m$. Then we obtain $N(k) \leqslant(\mu+2) k$. Indeed, we have $a^{-k} b^{n^{k}} a^{k}=b^{m^{k}}$, and the exponent of $p$ in the factorization of $m^{k}$ is at most $(\mu+1) k$.

The triple $\left(x_{k}=a, y_{k}=b^{n^{k}}, z_{k}=b\right)$ is a root of $w_{i}$ if and only if $i \leqslant N(k)$. Indeed:

- if $i \leqslant N(k)$, then $a^{-i} b^{n^{k}} a^{i}$ is a power of $b$, so that $\left[a^{-i} b^{n^{k}} a^{i}, b\right]=1$;

- if $i>N(k)$, then

$$
\left[a^{-i} b^{n^{k}} a^{i}, b\right]=a^{-(i-N(k))} b^{\alpha(k)} a^{i-N(k)} \cdot b \cdot a^{-(i-N(k))} b^{-\alpha(k)} a^{i-N(k)} \cdot b^{-1} .
$$

This is reduced in $\operatorname{BS}(m, n)$, since $|m|>1$ and $n$ does not divide $\alpha(k)$.

\footnotetext{
${ }^{2}$ By theorems of Mal'cev and Auslander [Seg83], Chapters 2 and 3, a finitely generated soluble group is linear over $\mathbb{Z}$ if and only if it is polycyclic.

${ }^{3}$ It is possible to use only one variable: replace $W$ by $W^{\prime}=\left\{\left[a^{-i} y a^{i}, b\right]: i \in \mathbb{N} \backslash\{0\}\right\} \subseteq$ $\mathrm{BS}(m, n) * \mathbb{F}(y)$.
} 
If we now consider a finite subset $W_{f}=\left\{w_{i_{1}}, \ldots, w_{i_{s}}\right\} \subset W$, then, choosing $k$ large enough, we have $N(k) \geqslant i_{1}, \ldots, i_{s}$. Consequently, the triple $\left(x_{k}, y_{k}, z_{k}\right)$ is in $\operatorname{Root}\left(W_{f}\right) \backslash \operatorname{Root}(W)$. This proves that $\mathrm{BS}(m, n)$ is not equationally noetherian.

Proof of Proposition 5.17. If $|m|=1$, one has $\overline{\mathrm{BS}}(m, \xi)=\mathbb{Z} \imath \mathbb{Z}$, which is equationally noetherian (e.g. it is linear over the field $\mathbb{Q}(X)$ ).

Let us now assume that $|m|>1$. Consider a sequence $\left(\xi_{n}\right)$ of rational integers such that $\left|\xi_{n}\right| \rightarrow \infty$ and $\xi_{n} \rightarrow \xi$ in $\mathbb{Z}_{m}$ for $n \rightarrow \infty$. We may assume that $|m|<\left|\xi_{n}\right|$ for all $n$. Set $W=\left\{w_{i}:=\left[x^{-i} y x^{i}, z\right] \mid i \in \mathbb{N} \backslash\{0\}\right\}$ and $\left(x_{k}=a, y_{k}=\right.$ $a^{k} b^{m^{k}} a^{-k}, z_{k}=b$ ), as in the proof of Proposition 5.18(2). We have proved the existence of natural numbers $N_{n}(k)$ such that $\left(x_{k}, y_{k}, z_{k}\right)$ is a root of $w_{i}$ in $\operatorname{BS}\left(m, \xi_{n}\right)$ if and only if $i \leqslant N_{n}(k)$. Moreover, Remark 5.19 gives the estimates

$$
k \leqslant N_{n}(k) \leqslant(\mu(m)+2) k
$$

for all $n$. Therefore, if we take a finite subset $W_{f}=\left\{w_{i_{1}}, \ldots, w_{i_{s}}\right\} \subset W$, then, choosing $k$ large enough, we have $N_{n}(k) \geqslant k \geqslant i_{1}, \ldots, i_{s}$ for all $n$. Therefore, for all $j$, we have $w_{i_{j}}\left(x_{k}, y_{k}, z_{k}\right)=1$ in all groups $\operatorname{BS}\left(m, \xi_{n}\right)$, and, passing to the limit, we see that $\left(x_{k}, y_{k}, z_{k}\right)$ is a root of $W_{f}$ in the group $\overline{\mathrm{BS}}(m, \xi)$.

On the other hand, by considering $w_{i}$ with $i>(\mu(m)+2) k$, we see that $w\left(x_{k}, y_{k}, z_{k}\right) \neq 1$ in all $\mathrm{BS}\left(m, \xi_{n}\right)$. Hence, in $\overline{\mathrm{BS}}(m, \xi)$, the triple $\left(x_{k}, y_{k}, z_{k}\right)$ is not a root of $W$. This proves that $\overline{\mathrm{BS}}(m, \xi)$ is not equationally noetherian.

\section{Dimensions}

In this section we give the first non-trivial Hausdorff dimension estimates of a subspace of the space of marked groups on two generators. Let us recall that the map $\overline{\mathrm{BS}}_{m}: \mathbb{Z}_{m} \rightarrow \mathscr{E}_{2}, \xi \mapsto \overline{\mathrm{BS}}(m, \xi)$, is injective on $\mathbb{Z}_{m}^{\times}$, [GS08], Theorem 1. In order to estimate Hausdorff dimensions of the subspaces

$$
Z_{m}^{\times}=\overline{\mathrm{BS}}_{m}\left(\mathbb{Z}_{m}^{\times}\right)=\left\{\overline{\mathrm{BS}}(m, \xi) \mid \xi \text { is invertible in } \mathbb{Z}_{m}\right\},
$$

we will prove that the maps between $Z_{m}^{\times}$and $\mathbb{Z}_{m}^{\times}$satisfy Hölder conditions and then apply classical results about Hausdorff dimension. In this section we always assume that $m$ is a rational integer satisfying $|m| \geqslant 2$.

6.1. Distances between limits. The first step towards Hausdorff dimension estimates is to estimate the distance between groups $\overline{\mathrm{BS}}(m, \xi)$ and $\overline{\mathrm{BS}}\left(m, \xi^{\prime}\right)$ in terms in the $m$-adic distance between $\xi$ and $\xi^{\prime}$.

Theorem 6.1. Let $h \in \mathbb{N} \backslash\{0\}$ and $\xi, \xi^{\prime} \in \mathbb{Z}_{m}$ satisfying $d:=\operatorname{gcd}(m, \xi)=$ $\operatorname{gcd}\left(m, \xi^{\prime}\right)$. Setting $\hat{m}=m / d$, we have: 
(1) If $\overline{\mathrm{BS}}(m, \xi)$ and $\overline{\mathrm{BS}}\left(m, \xi^{\prime}\right)$ have the same relations up to length $2(|m|+1) h+$ $2|m|+6$, then $\xi \equiv \xi^{\prime}\left(\bmod \hat{m}^{h} d \mathbb{Z}_{m}\right)$;

(2) If $\xi \equiv \xi^{\prime}\left(\bmod \hat{m}^{h} d \mathbb{Z}_{m}\right)$, then $\overline{\mathrm{BS}}(m, \xi)$ and $\overline{\mathrm{BS}}\left(m, \xi^{\prime}\right)$ have the same relations up to length $2 h$.

Proof. Due to Corollary 2.10 we may work in $\widetilde{\mathrm{BS}}(m, \xi)$ and $\widetilde{\mathrm{BS}}\left(m, \xi^{\prime}\right)$ instead of $\overline{\mathrm{BS}}(m, \xi)$ and $\overline{\mathrm{BS}}\left(m, \xi^{\prime}\right)$. Recall that, given the free abelian groups of countable rank

$$
\begin{aligned}
E & =\mathbb{Z} e_{0} \oplus \mathbb{Z} e_{1} \oplus \mathbb{Z} e_{2} \oplus \cdots, \\
M & =\mathbb{Z} m e_{0} \oplus \mathbb{Z}\left(e_{1}-r_{1}(\xi) e_{0}\right) \oplus \mathbb{Z}\left(e_{2}-r_{2}(\xi) e_{0}\right) \oplus \cdots \leqslant E, \\
M^{\prime} & =\mathbb{Z} m e_{0} \oplus \mathbb{Z}\left(e_{1}-r_{1}\left(\xi^{\prime}\right) e_{0}\right) \oplus \mathbb{Z}\left(e_{2}-r_{2}\left(\xi^{\prime}\right) e_{0}\right) \oplus \cdots \leqslant E, \\
E_{1} & =\mathbb{Z} e_{1} \oplus \mathbb{Z} e_{2} \oplus \cdots \leqslant E,
\end{aligned}
$$

we have

$$
\begin{aligned}
\widetilde{\mathrm{BS}}(m, \xi) & \left.=\langle a, E| a \psi(x) a^{-1}=x \text { for all } x \in E_{1}\right\rangle, \\
\widetilde{\mathrm{BS}}\left(m, \xi^{\prime}\right) & \left.=\langle a, E| a \psi^{\prime}(x) a^{-1}=x \text { for all } x \in E_{1}\right\rangle,
\end{aligned}
$$

where the isomorphism $\psi: E_{1} \rightarrow M$ is defined by $\psi\left(e_{1}\right)=m e_{0}$ and $\psi\left(e_{i+1}\right)=$ $e_{i}-r_{i}(\xi) e_{0}$ for $i>0$, and the isomorphism $\psi^{\prime}: E_{1} \rightarrow M^{\prime}$ is defined similarly. Recall also that the element $b \in \overline{\mathrm{BS}}(m, \xi)$ corresponds to $e_{0} \in \widetilde{\mathrm{BS}}(m, \xi)$. By Proposition 2.6 , the condition $\xi \equiv \xi^{\prime}\left(\bmod \hat{m}^{h} d \mathbb{Z}_{m}\right)$ is equivalent to $r_{i}(\xi)=r_{i}\left(\xi^{\prime}\right)$ for $i=1, \ldots, h$. We will consider the latter condition.

(1) Let $w=w\left(m, r_{1}(\xi), \ldots, r_{h}(\xi)\right) e_{0} w\left(-m,-r_{1}(\xi), \ldots,-r_{h}(\xi)\right)\left(-e_{0}\right)$ be defined as in Lemma 5.6. Since $|w| \leq 2(|m|+1) h+2|m|+6$ and $w=1$ in $\widetilde{\operatorname{BS}}(m, \xi)$, we also have $w=1$ in $\widetilde{\mathrm{BS}}\left(m, \xi^{\prime}\right)$. We deduce from Lemma 5.6 that $r_{i}(\xi)=r_{i}\left(\xi^{\prime}\right)$ for $i=1, \ldots, h$.

(2) Let $w$ be a (freely reduced) word on the alphabet $\left\{a^{ \pm 1}, b^{ \pm 1}\right\}$ satisfying $|w| \leqslant 2 h$. By substituting occurrences of $b^{\alpha}$ by $\alpha e_{0}$, we obtain a sequence $s=$ $\left(x_{0}, a^{\varepsilon_{1}}, x_{1}, \ldots, a^{\varepsilon_{k}}, x_{k}\right)$, with $k \geq 0$, of length at most $2 h$, where $\varepsilon_{i}= \pm 1$ and $x_{i}$ is an element of the subgroup $\mathbb{Z} e_{0} \leqslant E$ for all $i$. What we have to show is that the product of the sequence $s$ vanishes in $\widetilde{\mathrm{BS}}(m, \xi)$ if and only if it vanishes in $\widetilde{\mathrm{BS}}\left(m, \xi^{\prime}\right)$.

We reduce the sequence $s$ in the HNN-extension $\widetilde{\mathrm{BS}}(m, \xi)$, that is, we perform, as long as possible, substitutions of:

- a subsequence $\left(a, x, a^{-1}\right)$, with $x \in M$, by the element $\psi^{-1}(x) \in E_{1}$;

- a subsequence $\left(a^{-1}, x, a\right)$, with $x \in E_{1}$, by the element $\psi(x) \in M$.

We then obtain a sequence $t=\left(y_{0}, a^{\delta_{1}}, y_{1}, \ldots, a^{\delta_{l}}, y_{l}\right)$, with $l \geqslant 0$, which is reduced in $\widetilde{\mathrm{BS}}(m, \xi)$ and whose product in the latter group is equal to the product of $s$. The number of substitutions from $s$ to $t$ is trivially at most $h$. Therefore, it is easy to see that $t$ and the intermediate sequences contain only $a^{ \pm 1}$ letters and elements of the subgroup $\mathbb{Z} e_{0} \oplus \cdots \oplus \mathbb{Z} e_{h}$.

Now we use the hypothesis $r_{i}(\xi)=r_{i}\left(\xi^{\prime}\right)$ for $i=1, \ldots, h$. Therefore, the relation

$$
M \cap\left(\mathbb{Z} e_{0} \oplus \cdots \oplus \mathbb{Z} e_{h}\right)=M^{\prime} \cap\left(\mathbb{Z} e_{0} \oplus \cdots \oplus \mathbb{Z} e_{h}\right)
$$


holds, and $\psi$ and $\psi^{\prime}$ are equal in restriction to $\mathbb{Z} e_{1} \oplus \cdots \oplus \mathbb{Z} e_{h+1}$. It is thus possible to reduce the sequence $s$ in $\widetilde{\mathrm{BS}}\left(m, \xi^{\prime}\right)$ by performing the same substitutions as in $\widetilde{\mathrm{BS}}(m, \xi)$. Hence, the sequences $s$ and $t$ have the same product in $\widetilde{\mathrm{BS}}\left(m, \xi^{\prime}\right)$. Moreover, the sequence $t$ is also reduced in $\widetilde{\mathrm{BS}}\left(m, \xi^{\prime}\right)$ - if not, an argument similar to the above one would show that $t$ is not reduced in $\widehat{\mathrm{BS}}(m, \xi)$.

Finally, by structure theorems on HNN-extensions, the product of $t$ vanishes in $\widetilde{\mathrm{BS}}(m, \xi)\left(\right.$ resp. $\left.\widetilde{\mathrm{BS}}\left(m, \xi^{\prime}\right)\right)$ if and only if $l=0$ and $y_{0}=0$ in $E$. This concludes the proof of part (2).

We now turn to the case $d=\operatorname{gcd}(m, \xi)=1$, that is, to the case of invertible $m$-adic integers. Recall that we chose the metric $d(\cdot, \cdot)$ on $\mathscr{E}_{2}$ given by $d\left(N_{1}, N_{2}\right)=$ $e^{-v\left(N_{1}, N_{2}\right)}$ if $N_{1} \neq N_{2}$, where $v\left(N_{1}, N_{2}\right)=\inf \left\{|w|: w \in N_{1} \triangle N_{2}\right\}$.

Corollary 6.2. Let $h \in \mathbb{N} \backslash\{0\}$ and $\xi, \xi^{\prime} \in \mathbb{Z}_{m}^{\times}$such that $\left|\xi-\xi^{\prime}\right|_{m}=|m|^{-h}$. Setting $x=\overline{\mathrm{BS}}(m, \xi)$ and $x^{\prime}=\overline{\mathrm{BS}}\left(m, \xi^{\prime}\right)$, we have

$$
e^{-2(|m|+1)(h+1)-2|m|-6} \leqslant d\left(x, x^{\prime}\right) \leqslant e^{-2 h-1} .
$$

Proof. If $d\left(x, x^{\prime}\right)<e^{-2(|m|+1)(h+1)-2|m|-6}$, then $\overline{\mathrm{BS}}(m, \xi)$ and $\overline{\mathrm{BS}}\left(m, \xi^{\prime}\right)$ have the same relations up to length $2(|m|+1)(h+1)+2|m|+6$. Theorem 6.1 (1) then gives $\left|\xi-\xi^{\prime}\right|_{m} \leqslant|m|^{-(h+1)}$.

On the other hand, the relation $\left|\xi-\xi^{\prime}\right|_{m}=|m|^{-h}$ implies that $\xi \equiv \xi^{\prime}\left(\bmod m^{h} \mathbb{Z}_{m}\right)$. Theorem 6.1 (2) then gives $d\left(x, x^{\prime}\right) \leqslant e^{-2 h-1}$.

6.2. Hausdorff dimension estimates. We set $f$ to be the inverse of the (bijective) map $\overline{\mathrm{BS}}_{m}: \mathbb{Z}_{m}^{\times} \rightarrow Z_{m}^{\times}$. We now show that $f$ and $f^{-1}=\overline{\mathrm{BS}}_{m}$ both satisfy a Hölder condition.

Proposition 6.3. For all $x, x^{\prime} \in Z_{m}^{\times}$, we have

$$
\left|f(x)-f\left(x^{\prime}\right)\right|_{m} \leqslant C d\left(x, x^{\prime}\right)^{\alpha},
$$

where $\alpha=(2(|m|+1))^{-1} \log |m|$ and $C$ is some positive constant.

Proof. Set $\xi=f(x)$ and $\xi^{\prime}=f\left(x^{\prime}\right)$, so that $x=\overline{\mathrm{BS}}(m, \xi)$ and $x^{\prime}=\overline{\mathrm{BS}}\left(m, \xi^{\prime}\right)$. Write $\left|\xi-\xi^{\prime}\right|_{m}=|m|^{-h}$ with $h \in \mathbb{N}$. Let us treat the case $h \in \mathbb{N} \backslash\{0\}$ first. Using Corollary 6.2 (at the second line), we get

$$
\begin{gathered}
\left|f(x)-f\left(x^{\prime}\right)\right|_{m}=\left|\xi-\xi^{\prime}\right|_{m}=e^{-h \log |m|}, \\
d\left(x, x^{\prime}\right) \geqslant e^{-2(|m|+1) h-4|m|-8}=C_{1} e^{-2(|m|+1) h}=C_{1}\left(e^{-h \log |m|}\right)^{\frac{2(|m|+1)}{\log |m|}},
\end{gathered}
$$

with $C_{1}>0$. Consequently, we have $d\left(x, x^{\prime}\right) \geqslant C_{1}\left|f(x)-f\left(x^{\prime}\right)\right|_{m}^{\alpha^{-1}}$, whence $\left|f(x)-f\left(x^{\prime}\right)\right|_{m} \leqslant C_{2} d\left(x, x^{\prime}\right)^{\alpha}$ for some $C_{2}>0$. 
Finally, in case $h=0$, that is, $\xi \not \equiv \xi^{\prime}(\bmod m)$, there is a word

$$
a^{2} b^{m} a^{-1} b^{-t} a^{-1} b \cdot a^{2} b^{-m} a^{-1} b^{t} a^{-1} b^{-1} \quad \text { with } 0 \leqslant t \leqslant|m|-1,
$$

which is trivial in one of the marked groups $x=\overline{\mathrm{BS}}(m, \xi), x^{\prime}=\overline{\mathrm{BS}}\left(m, \xi^{\prime}\right)$ but not in the other one. This gives a constant $D>0$ such that $d\left(x, x^{\prime}\right) \geqslant D$, hence a constant $C_{3}>0$ such that $\left|f(x)-f\left(x^{\prime}\right)\right|_{m}=1 \leqslant C_{3} d\left(x, x^{\prime}\right)^{\alpha}$.

Proposition 6.4. For all $\xi$, $\xi^{\prime} \in \mathbb{Z}_{m}^{\times}$, we have

$$
d\left(f^{-1}(\xi), f^{-1}\left(\xi^{\prime}\right)\right) \leqslant\left|\xi-\xi^{\prime}\right|_{m}^{\beta},
$$

where $\beta=2(\log |m|)^{-1}$.

Proof. Let us write $\left|\xi-\xi^{\prime}\right|_{m}=|m|^{-h}$ with $h \in \mathbb{N}$. By Corollary 6.2, we have $d\left(f^{-1}(\xi), f^{-1}\left(\xi^{\prime}\right)\right) \leqslant e^{-2 h-1}$ (note that for $h=0$ this is trivially true, since $\left.\operatorname{diam}\left(\mathcal{G}_{2}\right)=e^{-1}\right)$. Hence we get

$$
d\left(f^{-1}(\xi), f^{-1}\left(\xi^{\prime}\right)\right) \leqslant e^{-2 h}=\left(e^{-h \log |m|}\right)^{2(\log |m|)^{-1}}=\left|\xi-\xi^{\prime}\right|_{m}^{\beta},
$$

which concludes the proof.

Theorem 6.5. The Hausdorff dimension of $Z_{m}^{\times}$satisfies

$$
\frac{\log |m|}{2(|m|+1)} \leqslant \operatorname{dim}_{H}\left(Z_{m}^{\times}\right) \leqslant \frac{\log |m|}{2}
$$

( for all $m$ such that $|m| \geqslant 2$ ).

Proof. It is well known, and easy to show, that $\operatorname{dim}_{H}\left(\mathbb{Z}_{m}^{\times}\right)=1$ with respect to the metric chosen in Section 1.2. Set $\alpha=(2(|m|+1))^{-1} \log |m|$ and $\beta=2(\log |m|)^{-1}$, as in Propositions 6.3 and 6.4. Classical theory of Hausdorff dimension (see e.g. [Fal03], Proposition 2.3, or [Rog70], Theorem 29) and these propositions imply that $1 \leqslant$ $\alpha^{-1} \operatorname{dim}_{H}\left(Z_{m}^{\times}\right)$and $\operatorname{dim}_{H}\left(Z_{m}^{\times}\right) \leqslant \beta^{-1}$, hence the result.

Corollary 6.6. The Hausdorff dimension of $\boldsymbol{E}_{2}$ with respect to $d(\cdot, \cdot)$ satisfies $\operatorname{dim}_{H}\left(\mathscr{E}_{2}\right) \geqslant \log (2) / 6$. In particular, the Hausdorff dimension of $\mathscr{E}_{2}$ with respect to any Hölder equivalent metric does not vanish.

We have estimated the Hausdorff dimension of the subspaces $Z_{m}^{\times}$, which are homeomorphic to the Cantor set (provided that $|m| \geq 2$ ). But many interesting subspaces of $\mathscr{E}_{2}$, or $\mathscr{E}_{n}$, appeared in the literature, e.g:

- the Cantor set of Grigorchuk groups [Gri84], many such groups have intermediate growth; 
- the closure $\mathscr{H}_{n} \subseteq \mathscr{E}_{n}$ of non-elementary hyperbolic groups considered by Champetier [Cha00];

- the minimal Cantor subset of $\mathscr{E}_{3}$ constructed by Nekrashevych [Nek07].

The first-named author has proved that the box-counting dimension (and hence the Hausdorff dimension) of the set of Grigorchuk groups vanishes [Guy07]. So does the box-counting dimension of the set of Nekrashevych groups since they share similar contracting properties with the latter. In the case of hyperbolic groups, we do not know wether or not the Hausdorff dimension vanishes.

\section{Complexity of the word and conjugacy problems}

In this section we study isomorphism invariants of groups originating from language theory, namely the space complexity and the Turing degree of the word and conjugacy problems. Our results are inspired by the works for Grigorchuk [Gri84] and Garzon and Zalcstein [GZ91] on the word problem of Grigorchuk groups. First, we show that the space complexity of the word problem for $\overline{\mathrm{BS}}(m, \xi)$ is tightly related to the space complexity of the rational integer sequence $\left(r_{i}(\xi)\right)$ (Proposition 7.3). Secondly, we show that the conjugacy problem for $\overline{\mathrm{BS}}(m, \xi)$ is Turing reducible to the word problem for $\overline{\mathrm{BS}}(m, \xi)$ (Corollary 7.7). For the sake of simplicity, our emphasis is on the space complexity of the word problem. Analogs of Proposition 7.3 for time complexity and the conjugacy problem could be proved if one is prepared to more technicalities.

Space complexity. Let $\mathcal{A}$ be a set. We denote by $\mathcal{A}^{*}$ the set all strings (or words) on $\mathcal{A}$. Let $s \in \mathcal{A}^{*}$. We denote by $|s|_{\mathscr{A}}$ the string length of $s$, that is, the number of symbols of $\mathcal{A}$ in $s$. We may simply write $|s|$ when the underlying set is clearly given by the context. A set $L$ is a language if it is a subset of $\mathcal{A}^{*}$ for some finite set $\mathcal{A}$ called alphabet.

Let $G$ be a group and let $X$ be a finite generating set of $G$. We denote by $\operatorname{WP}(G, X)$ the set of strings $s \in\left(X \cup X^{-1}\right)^{*}$ such that $s=1$ in $G$, i.e., $s$ reduces to the trivial element of $G$. The decision problem of membership in $\operatorname{WP}(G, X)$ is called the word problem with respect to $X$. The Turing time and space complexity of the language $\mathrm{WP}(G, X)$ are group-theoretic properties independent of $X$ [MO85]; so $X$ will be omitted.

Nota Bene 7.1. A Turing machine $M$ is an off-line Turing machine if it has a read-only input tape with endmarkers and finitely many semi-infinite storage tapes. All Turing machines considered in this section are off-line Turing machines that halt on every input. We refer the reader to [HU79] for the complete definitions of terms used in this section. 
Let $\mathrm{M}$ be an off-line Turing machine and let $f: \mathbb{R}_{+} \rightarrow \mathbb{N}$ be a function. If for every input word of length $n$, the machine M scans at most $f(n)$ cells on any storage tape, then $\mathrm{M}$ is said to be an $f(n)$ space-bounded Turing machine. We denote by $\operatorname{DSPACE}(f)$ (resp. NSPACE$(f)$ ) the class of languages which are accepted by a deterministic (resp. non-deterministic) $f(n)$ space-bounded Turing machine. A language $L$ is recursive if it is accepted by a Turing machine. A function $g: \mathbb{N}^{k} \rightarrow \mathbb{N}^{l}$ is a recursive function if it can be computed by a Turing machine (the $k$ arguments $i_{1}, \ldots, i_{k}$ of $g$ are initially placed on the input tape separated by 1 's, as $0^{i_{1}} 10^{i_{2}} 1 \ldots 10^{i_{k}}$, the $l$ arguments are placed similarly in some output tape). A function $g: \mathbb{N} \rightarrow \mathbb{N}^{l}$ is said to belong to $\operatorname{DSPACE}(f)$ (resp. $\left.\operatorname{NSPACE}(f)\right)$ if there exists a deterministic (resp. non-deterministic) Turing machine taking as input the binary expansion of $j$ and computing $g(j)$ in space bounded above by $f(n)$ where $n$ is the number of binary digits of $j$. A language $L$ (resp. a function $g$ ) is said to separate the inclusion of two space complexity classes

\section{$\operatorname{DSPACE}(f) \subset \mathrm{NSPACE}(f)$}

if $L$ (resp. $g$ ) belongs to $\operatorname{NSPACE}(f)$ but not to $\operatorname{DSPACE}(f)$. Proofs below use of the Tape Compression Theorem [HU79], Theorem 12.1, without mentioning it: the equality of language classes

$$
\operatorname{DSPACE}(f)=\operatorname{DSPACE}(c f)
$$

holds for any $c>0$, with an analogue statement in the non-deterministic case.

Time complexity is analogously defined by counting the number of state transitions of a Turing machine with a read-and-write input tape. Every input word of length $n$ requires at least $n$ state transitions to be entirely read, hence $\operatorname{DTIME}(n)$ is the smallest time complexity class. For every function $f$, we have $\operatorname{DTIME}(f) \subset \operatorname{DSPACE}(f)$. We collect few facts on the word problem of finitely generated groups.

- The language $\operatorname{WP}(G)$ is regular if and only if $G$ is a finite group [Ani71]. If $G$ is infinite then $\mathrm{WP}(G)$ does not belong to DSPACE $(\log \log )$ [HS65].

- The language $\operatorname{WP}(G)$ is context-free if and only if $G$ is virtually free [MS83], [Dun85].

- The language $\operatorname{WP}(G)$ belongs to $\operatorname{DSPACE}(\log )$ if $G$ is a linear group over a field of characteristic zero [LZ77]. There exists a finitely presented non-linear group $G$ such that $\operatorname{WP}(G) \in \operatorname{DSPACE}(\log )$ [Waa81].

- There is no known example of a "simple" group presentation for which the word problem does not belong to DSPACE $(\log )$.

- If $G$ contains a copy of $\mathbb{Z}$ then $\operatorname{WP}(G)$ does not belong to $\operatorname{DSPACE}(g)$ for any $g$ such that $g(n) / \log (n)$ tends to 0 [AGM92], Theorem 2. In particular, log is a sharp bound for the space complexity of the word problem of any infinite finitely generated linear group.

- The word problem of a word hyperbolic group $G$ is solvable in real time [Hol00]. In particular $\mathrm{WP}(G) \in \operatorname{DTIME}(n)$. 
Let $p, q \in \mathbb{Z} \backslash\{0\}$ and let $\mathrm{WP}(p, q)$ (resp. in $\mathrm{WP}(m, \xi)$ ) be the set of strings $s \in\left\{a^{ \pm 1}, b^{ \pm 1}\right\}^{*}$ such that $s=1$ in $\operatorname{BS}(p, q)$ (resp. $\left.\overline{\mathrm{BS}}(m, \xi)\right)$. Given $\overline{\mathrm{BS}}(m, \xi)$, we define the function $r=r_{m, \xi}$ on $\mathbb{N}$ by

$$
r(0)=|m|, \quad r(n):=r_{n}\left(\epsilon_{m} \xi\right),
$$

where $\epsilon_{m}$ is the sign of $m$. This definition is motivated by the fact that $\operatorname{WP}(m, \xi)=$ $\mathrm{WP}\left(|m|, \epsilon_{m} \xi\right)$ since $\overline{\mathrm{BS}}(m, \xi)$ and $\overline{\mathrm{BS}}(-m,-\xi)$ are isomorphic as marked groups. The following proposition can be proved by using arguments similar to those of Lemma 7.4.

\section{Proposition 7.2. $\operatorname{WP}(p, q) \in \operatorname{DSPACE}(n) \cap \operatorname{DTIME}\left(n^{2}\right)$.}

As $\mathrm{BS}(p, q)$ is not virtually free, we observe that the language $\operatorname{WP}(p, q)$ is not a context-free language. The complement of $\operatorname{WP}(p, q)$ is not a context-free language either, except for $|p|=|q|[\mathrm{HRR}+05]^{4}$. Solvable Baumslag-Solitar groups (i.e., groups $\mathrm{BS}(p, q)$ with $|p|=1$ or $|q|=1)$ have a tidy real-time word problem [HR03], Theorem 2.1. We still ignore wether $\operatorname{WP}(p, q)$ belongs to DSPACE $(\log )$ in the case $\mathrm{BS}(p, q)$ is not linear. (Recall that $\mathrm{BS}(p, q)$ is linear if and only if either $|p|=|q|$ or $|p|=1$ or $|q|=1$ by Proposition 5.18.) The reader interested in geodesic languages of Baumslag-Solitar groups should consult [Eld05], [DL].

Provided that $r$ belongs to $\operatorname{DSPACE}(n)$, Proposition 7.2 holds for $\mathrm{WP}(m, \xi)$ and it corresponds to the lowest complexity bound we obtain. Our next result relate the space complexity of $\mathrm{WP}(m, \xi)$ to the space complexity of $r$. Let us stress on the fact that functions $r=r_{m, \xi}$ defined by (9) are "numerous" because of Proposition 2.5 (iii): for any $m \in \mathbb{Z} \backslash\{0\}, d \in \mathbb{N} \backslash\{0\}$, and for any $g: \mathbb{N} \rightarrow\{0, \ldots,|m|-1\}$ there is some $\xi \in \mathbb{Z}_{m}^{\times}$such that $g(n)=r_{n}\left(\epsilon_{m} \xi\right)$ for all $n \geq 2$. Hence the following proposition can be seen as a result of density in the space hierarchy.

Proposition 7.3. Let $f$ be a non-decreasing function such that $f(n) \geq n$ and $\operatorname{DSPACE}(f) \neq \operatorname{NSPACE}(f)$. Let $\overline{\mathrm{BS}}(m, \xi)$ be such that $r$ separates the inclusion $\operatorname{DSPACE}(f) \subset \operatorname{NSPACE}(f)$. Then $\mathrm{WP}(m, \xi)$ separates the inclusion

$$
\operatorname{DSPACE}(f(n / 6|m|)) \subset \operatorname{NSPACE}(f(n)) \text {. }
$$

This result is an immediate consequence of the following two lemmas.

Lemma 7.4. Assume $r \in \operatorname{DSPACE}(f(n))$ for some non-decreasing function $f$. Then $\operatorname{WP}(m, \xi) \in \operatorname{DSPACE}(n+f(n))$. Likewise for NSPACE.

Lemma 7.5. Assume $\mathrm{WP}(m, \xi) \in \operatorname{DSPACE}(f(n / 6|m|))$ for some non-decreasing function $f$ such that $f(n) \geq n$. Then $r \in \operatorname{DSPACE}(f(n))$. Likewise for NSPACE.

\footnotetext{
${ }^{4}$ It is incorrectly claimed in the proof of $[\mathrm{HRR}+05]$, Theorem 13 , that $|p|=|q|$ if and only if $\operatorname{BS}(p, q)$ is virtually abelian. The condition $|p|=|q|$ is less restrictive for it means that $\operatorname{BS}(p, q)$ contains a copy of the direct product $\mathbb{F}_{|p|} \times \mathbb{Z}$ as a finite index subgroup, or equally that $\operatorname{BS}(p, q)$ is linear over $\mathbb{Z}$.
} 
Let us summarize the idea of the proof of Lemma 7.4. Applying to a given word $w \in\left\{a^{ \pm 1}, b^{ \pm 1}\right\}^{*}$ the natural algorithm originating from Britton's lemma, we obtain a reduced sequence for $w$. This reduction is carried out within at most $|w|_{a}$ steps and at each step we consider a word whose length is at most $|m|$ times the length of the previous one. As we encode the exponents of $a$ and $b$ by means of their binary expansions, this stretching factor becomes an additive constant which explains the linear part of the space complexity bound. The other part of the bound comes from the fact that we need to compute $r(n)$ to reduce words $w$ such that $|w|_{a}=n$.

As for the proof of Lemma 7.5, we notice that a Turing machine which can solve the word problem for $\overline{\mathrm{BS}}(m, \xi)$, can decide which of the words defined in Lemma 5.6 are trivial. Hence it can be used to compute $r_{i}(\xi)$ for every $i$.

We will work with our favoured HNN extension $\widetilde{\mathrm{BS}}(m, \xi)$ instead of $\overline{\mathrm{BS}}(m, \xi)$. In order to make a careful enough counting of the numbers of scanned cells, we will use following notations. We fix $\mathcal{A}:=\left\{a^{ \pm 1}, \pm e_{0}, \pm e_{1}, \ldots\right\}$. Let $m \in \mathbb{Z} \backslash\{0\}$ and $\xi \in \mathbb{Z}_{m}$. Given $w \in \mathcal{A}^{*}$, we can rewrite $w$ in $\langle a\rangle * E$ under the form

$$
w^{(0)}=a^{\alpha_{1}} c_{1} a^{\alpha_{2}} c_{2} \ldots a^{\alpha_{h}} c_{h}
$$

with $c_{j}=\left(\beta_{0 j} e_{0}\right)\left(\beta_{1 j} e_{1}\right) \ldots\left(\beta_{k_{j} j} e_{k_{j}}\right), \alpha_{j}, \beta_{l j} \in \mathbb{Z}$ for all $l, j$.

We denote by $\varepsilon_{j}$ the sign of $\alpha_{j}$. We suppose that the following holds: there is some $j$ such that

$$
\varepsilon_{j}=-\varepsilon_{j+1}=-1 \text { and } c_{j} \in E_{m, \xi} \quad \text { or } \quad \varepsilon_{j}=-\varepsilon_{j+1}=1 \text { and } c_{j} \in E_{1} \text {. }
$$

We denote by $\ell=\ell(w)$ the smallest $j$ such that $(*)$ holds. Let $w^{\prime}$ be the word we get from $w^{(0)}$ by replacing $a^{\alpha_{\ell}} c_{\ell} a^{\alpha_{\ell+1}}$ by $a^{\alpha_{\ell}-\varepsilon_{\ell}} \phi^{\varepsilon_{\ell}}\left(c_{\ell}\right) a^{\alpha_{\ell+1}+\varepsilon_{\ell}}$ in $w$ and reducing this new word as in (10). We write $w^{\prime}=a^{\alpha_{1}^{\prime}} c_{1}^{\prime} a^{\alpha_{2}^{\prime}} \ldots a^{\alpha_{h^{\prime}}^{\prime}} c_{h^{\prime}}^{\prime}$. Notice that a given exponent $\alpha$ of $a$ in $w$ either remains unchanged in $w^{\prime}$, vanishes or is replaced by some $\alpha^{\prime}$ such that $\left|\alpha^{\prime}\right|=|\alpha|-1$. The subwords $c_{j}$ remain unchanged in $w^{\prime}$ or vanish, except one which is replaced by some subword $c^{\prime}$ with $\left|c^{\prime}\right| \leq(2+|m|) n$ where $n=|w|$. As long as $(*)$ holds for $w^{(i)}=a^{\alpha_{1}^{(i)}} c_{1}^{(i)} a^{\alpha_{2}^{(i)}} \ldots a^{\alpha_{h^{(i)}}^{(i)}} c_{h^{(i)}}^{(i)}$ with $i \geq 0$, we can define $w^{(i+1)}=\left(w^{(i)}\right)^{\prime}$.

By Britton's lemma, for any $w \in \mathcal{A}^{*}$, there is some $i=i(w) \geq 0$ such that $w^{(i)}=1$ is a reduced form for $w$. We call the previous algorithm the Britton's algorithm.

Proof of Lemma 7.4. By hypothesis, there is an $f(n)$ space-bounded Turing machine $\mathrm{M}_{r}$ computing $r(n)$. We denote by $R_{1}$ its input tape and by $R_{k}(2 \leq k \leq p)$ its storage tapes. We design an off-line Turing machine $M$ that halts on every input $w \in\left\{a^{ \pm 1}, \pm e_{0}\right\}^{*}$ : if a non-trivial reduced form for $w$ has been found, it halts without accepting, else $w$ is reduced to 1 and $\mathrm{M}$ halts in an accepting state. Tape $\mathrm{I}$ is the read-only input tape where $w$ is displayed without accounting for any space. At the beginning, $\mathrm{M}$ writes the $\operatorname{string} s^{(0)}$ on tape 0 that encodes $w^{(0)}$ :

$$
s^{(0)}:=\varnothing \varepsilon_{1} \bar{\alpha}_{1} \bar{c}_{1} \varepsilon_{2} \bar{\alpha}_{2} \bar{c}_{2} \ldots \varepsilon_{h} \bar{\alpha}_{h} \bar{c}_{h} \$ \text {. }
$$


The strings $\bar{\alpha}_{j} \in\{0,1\}^{*}$ are the binary expansions of $\left|\alpha_{j}\right|$; each string $\bar{c}_{j} \in\{0,1, \pm\}^{*}$ is the concatenation of the binary expansions of the numbers $\left|\beta_{l j}\right|$ separated by sign symbols. If $\alpha_{1}=0$ (respectively $c_{h}=0$ ) then $\varepsilon_{1} \bar{\alpha}_{1}$ (respectively $\bar{c}_{h}$ ) is replaced by the empty string.

We now describe how $\mathrm{M}$ works on its storage tapes $R_{k}(1 \leq k \leq p), T_{0}, T_{1}$ and D. First, the machine read the input: while the head of tape I scans the first $j$ symbols of $w$, M stores the number $j$ using a counter situated in tape $R_{1}$ and then $\mathrm{M}$ computes and stores $r_{j}\left(\epsilon_{m} \xi\right)$ in some of the tapes $R_{k}$ by simulating $M_{r}$. Meanwhile, the head of tape $T_{0}$ writes $s^{(0)}$, following an obvious linearly space-bounded algorithm. Once the input is read, M goes ahead by running Britton's algorithm. During the $i$-th step of this algorithm, with $i$ even, the head of $T_{0}$ writes the string $s^{(i)}$ encoding $w^{(i)}$ over $s^{(i-2)}$ if condition $(*)$ holds for $w^{(i-1)}$. The latter word is encoded by a string $s^{(i-1)}$ stored in tape $T_{1}$. In the next step, the head of tape $T_{1}$ writes the string $s^{(i+1)}$ encoding $w^{(i+1)}$ over $s^{(i-1)}$ if condition $(*)$ holds for $w^{(i-1)}$. Tape $\mathrm{D}$ is a draft tape used to carry out two kind of arithmetical computations on binary expansions: the tests for condition $(*)$ and the computations of $c^{(i)}$. The content of tape $\mathrm{D}$ is erased after each step. The machine $\mathrm{M}$ halts in a state of acceptation if $s^{(i)}$ is the trivial string. It halts without accepting in case condition $(*)$ does not hold for $w^{(i)}$.

Space bound. The machine M scans at most $f(n)$ cells on the storage tapes $R_{k}$ while computing $r_{1}\left(\epsilon_{m} \xi\right), \ldots, r_{n}\left(\epsilon_{m} \xi\right)$. It also scans at most $C_{0} n$ cells while storing each number $j$ and all numbers $r_{j}\left(\epsilon_{m} \xi\right)$ for $j \leq n$, where $C_{0}>0$ is independent of $n$.

Since $\left|s^{(i+1)}\right| \leq\left|s^{(i)}\right|+\log _{2}(|m|)$ and $\left|s^{(0)}\right| \leq 2 n+2$, we deduce that $\mathrm{M}$ scans at most $C_{1} n$ cells on the storage tapes $T_{0}$ and $T_{1}$, where $C_{1}>0$ is independent of $n$. In order to decide if $c_{j}^{(i)}$ belongs to $E_{m, \xi}$ or $E_{1}$, M uses the formula of Proposition 2.9 (iii): according to the signs of $\alpha_{j}^{(i)}$ and $\alpha_{j+1}^{(i)}$, M carries out the division of $\left.\gamma_{m}:=\beta_{0 j}^{(i)}+\beta_{1 j}^{(i)} r_{1}\left(\epsilon_{m} \xi\right)+\beta_{2 j}^{(i)} r_{2}\left(\epsilon_{m} \xi\right)+\cdots+\beta_{k_{j} j}^{(i)} r_{k_{j}}\left(\epsilon_{m} \xi\right)\right)$ by $|m|$ or divides $\gamma_{\xi}:=\beta_{1 j}^{(i)}+\beta_{2 j}^{(i)} r_{1}\left(\epsilon_{m} \xi\right)+\beta_{2 j}^{(i)} r_{2}\left(\epsilon_{m} \xi\right)+\cdots+\beta_{k_{j} j}^{(i)} r_{k_{j}}\left(\epsilon_{m} \xi\right)$ by $|m|$ if moreover $\beta_{0 j}^{(i)}=0$. As $\log _{2}(1+|\gamma|) \leq\left|s^{(i+1)}\right|$, for $\gamma=\gamma_{m}$, $\gamma_{\xi}$, this requires to scan at most $C_{2} n$ cells on tape $\mathrm{D}$, where $C_{2}>0$ is independent of $n$. In order to compute $c^{(i)}$, no more than $\left|s^{(i+1)}\right|$ cells need to be scanned on tape D. Hence the number of cells scanned by $\mathrm{M}$ on tape $\mathrm{D}$ is linearly bounded. All in all, we get $\mathrm{WP}(m, \xi) \in \operatorname{DSPACE}(n+f(n))$.

The first part of the proof of Lemma 7.5 is based on the following facts.

Lemma 7.6. Let $m, n \in \mathbb{Z} \backslash\{0\}$ with $|m|>1$ and let $\xi \in \mathbb{Z}_{m}$. Let $v_{k}=\left[a b^{k} a^{-1}, b\right]$ for $k \in \mathbb{Z}$. Then we have: $v_{k}=1$ in $\overline{\mathrm{BS}}(m, \xi)$ if and only if $k \equiv 0(\bmod m \mathbb{Z})$.

Proof. Let $|n|>1$. We deduce from Britton's lemma the following claim: for every $k \in \mathbb{Z}$, we have $v_{k}=1$ in $\operatorname{BS}(m, n)$ if and only if $k \equiv 0(\bmod m \mathbb{Z})$. As $\operatorname{BS}\left(m, \xi_{n}\right)$ 
tends to $\overline{\mathrm{BS}}(m, \xi)$ as $n$ goes to infinity, $v_{k}$ is trivial in $\overline{\mathrm{BS}}(m, \xi)$ if and only if it is trivial in $\mathrm{BS}\left(m, \xi_{n}\right)$ for all $n$ large enough, which completes the proof.

For $h \geq 1, t_{1}, \ldots, t_{h} \in\{0, \ldots,|m|-1\}$, we set

$$
v\left(|m|, t_{1}, \ldots, t_{h}\right):=w\left(|m|, t_{1}, \ldots, t_{h}\right) b w\left(-|m|,-t_{1}, \ldots,-t_{h}\right) b^{-1},
$$

where $w\left(|m|, t_{1}, \ldots, t_{h}\right)$ is defined as in Lemma 5.6.

Proof of Lemma 7.5. By hypothesis, there is a deterministic $f(n / 6|m|)$ spacebounded Turing machine $\mathrm{M}$ that solves the word problem for $\overline{\mathrm{BS}}(m, \xi)$. We design a Turing machine $\mathrm{M}^{\prime}$ computing $r(n)$ as follows. The storage tapes of $\mathrm{M}^{\prime}$ consists of the tapes of $\mathrm{M}$ and two other tapes $\mathrm{W}$, and $\mathrm{O}$ (output tape). The tape $\mathrm{W}$ identifies with the input tape of $\mathrm{M}$ and $\mathrm{M}^{\prime}$ simulates $\mathrm{M}$ on every tape of $\mathrm{M}$.

Computation of $|m|$. By Lemma 7.6, we have $|m|=\min \left\{k \geq 1 \mid v_{k}=1\right.$ in $\overline{\mathrm{BS}}(m, \xi)\}$. The machine $\mathrm{M}^{\prime}$ first writes $v_{k}$ on tape $\mathrm{W}$ for $k=1$ and runs $\mathrm{M}$. While $v_{k}$ is not accepted by $\mathrm{M}$, the machine $\mathrm{M}^{\prime}$ writes $v_{k+1}$ over $v_{k}$, adds one to a counter storing $k$ in tape $\mathrm{O}$ and clears the storage tapes of $\mathrm{M}$. If $v_{k}$ is accepted, which means $k=|m|$, then $\mathbf{M}^{\prime}$ clears tape $\mathrm{W}$.

Computation of $r_{n}\left(\epsilon_{m} \xi\right)$. Using two counters which store $i \leq n$ and $t \in\{0, \ldots,|m|-1\}$ in tape $\mathrm{O}$, the machine $\mathrm{M}^{\prime}$ lists recursively the words $w_{i}(t)=$ $v\left(|m|, r_{1}\left(\epsilon_{m} \xi\right), \ldots, r_{i-1}\left(\epsilon_{m} \xi\right), t\right)$ on tape $\mathrm{W}$. Once a word $w_{i}(t)$ is written on tape $\mathrm{W}$, the machine $\mathrm{M}^{\prime}$ runs $\mathrm{M}$. If $w_{i}(t)$ is not accepted by $\mathbf{M}$, then $\mathbf{M}^{\prime}$ writes $w_{i}(t+1)$ over $w_{i}(t)$, clears the storage tapes of $\mathrm{M}$ and runs $\mathrm{M}$ again. If the word written on $W$ is accepted by $\mathrm{M}$, which means $t_{i}=r_{i}\left(\epsilon_{m} \xi\right)$ by Lemma 5.6, then $\mathrm{M}^{\prime}$ stores $r_{i}(\xi)$ in tape $\mathrm{O}$ and restarts with $w_{i+1}(0)$ or halts if $i=n$.

Space bound. Obviously the number of cells scanned by $\mathbf{M}^{\prime}$ to compute $|m|$ is bounded by some constant $C(m)$ independent of $n$. The number of cells scanned by $\mathrm{M}^{\prime}$ while writing words $v\left(|m|, t_{1}, \ldots, t_{n}\right)$ on tape $\mathrm{W}$ is bounded by $\left|v\left(|m|, t_{1}, \ldots, t_{n}\right)\right| \leq$ $6 n|m|$, the number of cells used to store $r_{1}(\xi), \ldots, r_{n}(\xi)$ is bounded by $n \log _{2}(|m|+1)$ and the number of cells scanned by $\mathbf{M}^{\prime}$ while simulating $\mathrm{M}$ over its storage tapes is bounded by $f(n)$. Hence $\operatorname{WP}(m, \xi) \in \operatorname{DSPACE}(f)$.

Turing degree. Let $E, F$ be languages. The language $E$ is said to be Turing reducible to $F$ if there is Turing machine $M$ with oracle $F$ whose accepted language is $E$. The language $E$ is said Turing equivalent to $F$ if $E$ is Turing reducible to $F$ and $F$ is Turing reducible to $E$. The Turing degree of $E$ (also called the degree of unsolvability of $E$ ) is the class all languages that are Turing equivalent to $E$. Let $f: E \rightarrow F$ be a function. We define the Turing degree of $f$ as the Turing degree of the graph of $f$. We denote by $C P(m, \xi)$ the set of pairs $\left(w, w^{\prime}\right) \in\left\{a^{ \pm 1}, b^{ \pm 1}\right\}^{*} \times\left\{a^{ \pm 1}, b^{ \pm 1}\right\}^{*}$ such that $w$ is conjugated to $w^{\prime}$ in $\overline{\mathrm{BS}}(m, \xi)$. We call the Turing degrees of $\mathrm{WP}(m, \xi), C P(m, \xi)$ the Turing degrees of the word problem and the conjugacy problem for $\overline{\mathrm{BS}}(m, \xi)$. These Turing degrees does not depend on the choice of a generating set for $\overline{\mathrm{BS}}(m, \xi)$. 
Corollary 7.7. The following Turing degrees coincide:

- the Turing degree of the word problem for $\overline{\mathrm{BS}}(m, \xi)$;

- the Turing degree of the conjugacy problem for $\overline{\mathrm{BS}}(m, \xi)$;

- the Turing degree of $r$.

In particular, the word problem is solvable for $\overline{\mathrm{BS}}(m, \xi)$, i.e., $\mathrm{WP}(m, \xi)$ is a recursive language if and only if $r$ is a recursive function.

In contrast, Britton has proved that the conjugacy problem for any HNN extension with base a finitely generated abelian group is solvable, i.e., both Turing degrees are $\mathbf{0}$ [Bri79]. The Turing degrees of the word and conjugacy problems need not be equal in general, as shows the optimal result of Miller: for every pair of recursively enumerable Turing degrees $\mathbf{a}, \mathbf{b}$ where $\mathbf{a}$ is Turing reducible to $\mathbf{b}$, there is a finitely presented group whose word problem has Turing degree a and whose conjugacy problem has Turing degree $\mathbf{b}$ [Mil71].

Observe that one can define recursive $m$-adic numbers in the very same way one defines recursive (equivalently computable) real numbers (see [Wei00] for a definition of computable real numbers). The Turing degree of an $m$-adic number is then defined by means of its Hensel expansion. If $\xi \in \mathbb{Z}_{m}^{\times}$, Corollary 7.7 then says that the word problem is solvable in $\overline{\mathrm{BS}}(m, \xi)$ if and only if $\xi$ is a recursive number and that the Turing degree of the word problem coincides with the Turing degree of $\xi$.

Proof. The last claim directly follows from Lemmas 7.4 and 7.5. From the proofs of these lemmas, we can easily deduce that the Turing degree of WP $(m, \xi)$ coincides with the Turing degree of $r$.

To complete the proof we design quite informally a Turing machine with oracle $r$ that solves the conjugacy problem in $\overline{\mathrm{BS}}(m, \xi)$. We fix the set of representatives $T_{m, \xi}=\left\{0, e_{0}, \ldots,(m-1) e_{0}\right\}$ of the cosets of $E_{1}$ in $E$ and the set of representatives $T_{1}=\left\{0, e_{0}, 2 e_{0}, \ldots\right\}$ of the cosets of $E_{m, \xi}$ in $E$. If $r$ can be computed by means of a Turing machine, Britton's algorithm (see the proof of Lemma 7.4) yields a reduced form in $\widetilde{\mathrm{BS}}(m, \xi)$ of any $w \in\left\{a^{ \pm 1}, \pm e_{0}\right\}^{*}$. The process of working from the right with the relations $a\left(m e_{0}\right) a^{-1}=e_{1}$ and $a\left(e_{i}-r_{i}(\xi) e_{0}\right) a^{-1}=e_{i+1}$ yields a normal form for $w$ with respect to the sets of representatives $T_{m, \xi}$ and $T_{1}$. Thus we can design a Turing machine with oracle $r$ that computes normal forms $\tilde{v}, \tilde{w}$ of cyclically reduced conjugates of $v$ and $w$ for any $v, w \in\left\{a^{ \pm 1}, \pm e_{0}\right\}^{*}$. If $|\tilde{v}|_{a} \neq|\tilde{w}|_{a}$, we deduce from Collin's lemma [LS77], Theorem 2.5, that $v$ is not a conjugate of $w$ in $\widetilde{\mathrm{BS}}(m, \xi)$. The machine can be designed in such a way that it halts in this case in a non-accepting state. Hence we can assume that $|\tilde{v}|_{a}=|\tilde{w}|_{a}$. Comparing the normal form $\tilde{v}$ to the normal form of each cyclic permutation of $\tilde{w}$, the machine can decide wether or not there exist, $e \in E$ and some cyclic permutation $(\tilde{w})^{*}$ of $\tilde{w}$ such that $\tilde{v}=e(\tilde{w})^{*}(-e)$. By Collin's lemma, it is enough to decide wether $v$ is a conjugate of $w$, provided either $|\tilde{v}|_{a}$ or $|\tilde{w}|_{a}$ is not zero. Hence we can assume that $v, w$ have their images in $E$. We deduce from Lemma 4.5 that $v$ is a conjugate of $w$ in $\widetilde{\mathrm{BS}}(m, \xi)$ if and only if 
there is some $n \in \mathbb{Z}$ such that $v=a^{n} w a^{-n}$ in $\widetilde{\mathrm{BS}}(m, \xi)$. Identifying $E$ with $B$ in Proposition 2.9 (i), we can consider $n(v, w)=\operatorname{deg} P_{v}(X)-\operatorname{deg} P_{w}(X)$. By means of a Turing machine with oracle $r$, we can compare the Laurent polynomials $P_{v}(X)$ and $X^{n(v, w)} P_{w}(X)$ and hence decide wether or not $v$ is a conjugate of $w$.

Remark 7.8. We can construct a family of public-key cryptosystems based on the word problems of limits of Baumslag-Solitar groups by adapting the construction in [GZ91] based on Grigorchuk groups. The attack conceived in $\left[\mathrm{GHM}^{+} 04\right]$ does not threaten these new cryptosystems since such an attack would require in our case at least $|m|^{N}$ numbers of computations when the length of the public-key is $N$, if we follow the cryptanalysis of the authors. However, another attack conceived in $\left[\mathrm{GHM}^{+} 04\right]$, namely the reaction attack against the Magyarik-Wagner protocol, can be proved to be successful.

Acknowledgments. We are very grateful to the referee for her, or his, thorough and dedicated work. We thank Yves de Cornulier and Pierre de la Harpe for their helpful comments on previous versions of this article. We also thank Nicolas Bartholdi for useful discussions concerning Section 2.1.

\section{References}

[AGM92] H. Alt, V. Geffert, and K. Mehlhorn, A lower bound for the nondeterministic space complexity of context-free recognition. Inform. Process. Lett. 42 (1992), 25-27. Zbl 0780.68081 MR 1160441

[Ani71] A. V. Anisimov, The group languages. Kibernetika 1971 (Kiev) (1971), no. 4, 18-24. Zbl 0241.68034 MR 0301981

$\left[\mathrm{ABL}^{+}{ }^{05}\right]$ G. N. Arzhantseva, J. Burillo, M. Lustig, L. Reeves, H. Short, and E. Ventura, Uniform non-amenability. Adv. Math. 197 (2005), 499-522. Zbl 1077.43001 MR 2173843

[Bau81] A. Baudisch, Subgroups of semifree groups. Acta Math. Acad. Sci. Hungar. 38 (1981), 19-28. MR 634562

[BMR99] G. Baumslag, A. Myasnikov, and V. Remeslennikov, Algebraic geometry over groups. I. Algebraic sets and ideal theory. J. Algebra 219 (1999), 16-79. Zbl 0938.20020 MR 1707663

[BMR97] G. Baumslag, A. Myasnikov, and V. Roman'kov, Two theorems about equationally Noetherian groups. J. Algebra 194 (1997), 654-664. Zbl 0888.20017 MR 1467171

[BS62] G. Baumslag and D. Solitar, Some two-generator one-relator non-Hopfian groups. Bull. Amer. Math. Soc. 68 (1962), 199-201. Zbl 0108.02702 MR 0142635

[BH86] E. Bédos and P. de la Harpe, Moyennabilité intérieure des groupes: définitions et exemples. Enseign. Math. (2) 32 (1986), 139-157. Zbl 0605.43002 MR 850556 
[Bri79] J. L. Britton, The conjugacy problem for an HNN extension of an abelian group. Math. Sci. 4 (1979), 85-92. Zbl 0415.20027 MR 561995

[Bry77] R. M. Bryant, The verbal topology of a group. J. Algebra 48 (1977), 340-346. Zbl 0408.20022 MR 0453878

[Cha50] C. Chabauty, Limite d'ensembles et géométrie des nombres. Bull. Soc. Math. France 78 (1950), 143-151. Zbl 0039.04101 MR 0038983

[Cha00] C. Champetier, L'espace des groupes de type fini. Topology 39 (2000), 657-680. Zbl 0959.20041 MR 1760424

[CG05] C. Champetier and V. Guirardel, Limit groups as limits of free groups. Israel J. Math. 146 (2005), 1-75. Zbl 1103.20026 MR 2151593

[Cor09a] Y. Cornulier, On the Cantor-Bendixson rank of metabelian groups. Ann. Inst. Fourier (Grenoble) 61 (2011), 593-618. Zbl 1238.20049 MR 2895067

[Cor09b] Y. Cornulier, A sofic group away from amenable groups. Math. Ann. 350 (2011), 269-275. Zbl 05903689 MR 2794910

[CGP07] Y. de Cornulier, L. Guyot, and W. Pitsch, On the isolated points in the space of groups. J. Algebra 307 (2007), 254-277. Zbl 1132.20018 MR 2278053

[Dav96] K. R. Davidson, $C^{*}$-algebras by example. Fields Inst. Monogr. 6, Amer. Math. Soc., Providence, RI, 1996. Zbl 0958.46029 MR 1402012

[DL] V. Diekert and J. Laun, On computing geodesics in Baumslag-Solitar groups. Internat. J. Algebra Comput. 21 (2011), 119-145. Zbl 1235.20041 MR 2787455

[Dun85] M. J. Dunwoody, The accessibility of finitely presented groups. Invent. Math. 81 (1985), 449-457. Zbl 0572.20025 MR 807066

[Eff75] E. G. Effros, Property $\Gamma$ and inner amenability. Proc. Amer. Math. Soc. 47 (1975), 483-486. Zbl 0321.22011 MR 0355626

[Eld05] M. Elder, A context-free and a 1-counter geodesic language for a BaumslagSolitar group. Theoret. Comput. Sci. 339 (2005), 344-371. Zbl 1142.68041 MR 2142503

[Fal03] K. Falconer, Fractal geometry. 2nd ed., John Wiley, Chichester 2003. Zbl 1060.28005 MR 2118797

[FM98] B. Farb and L. Mosher, A rigidity theorem for the solvable Baumslag-Solitar groups. Invent. Math. 131 (1998), 419-451. Zbl 0937.22003 MR 1608595

[FG06] A. Fel'shtyn and D. L. Gonçalves, Twisted conjugacy classes of automorphisms of Baumslag-Solitar groups. Algebra Discrete Math. 2006 (2006), no. 3, 36-48. Zbl 1116.20025 MR 2321932

[FG08] A. Fel'shtyn and D. L. Gonçalves, The Reidemeister number of any automorphism of a Baumslag-Solitar group is infinite. In Geometry and dynamics of groups and spaces, Progr. Math. 265, Birkhäuser, Basel 2008, 399-414. Zbl 1138.20033 MR 2402411

[FH94] A. Fel'shtyn and R. Hill, The Reidemeister zeta function with applications to Nielsen theory and a connection with Reidemeister torsion. K-Theory 8 (1994), 367-393. Zbl 0814.58033 MR 1300546 
[GZ91] M. Garzon and Y. Zalcstein, The complexity of Grigorchuk groups with application to cryptography. Theoret. Comput. Sci. 88 (1991), 83-98. Zbl 0749.68040 MR 1130373

[GG08] T. Gelander and Y. Glasner, Countable primitive groups. Geom. Funct. Anal. 17 (2008), 1479-1523. Zbl 1138.20005 MR 2377495

[Gil79] D. Gildenhuys, Classification of soluble groups of cohomological dimension two. Math. Z. 166 (1979), 21-25. Zbl 0414.20032 MR 526863.

$\left[\mathrm{GHM}^{+}{ }^{04}\right]$ M. I. González Vasco, D. Hofheinz, C. Martínez, and R. Steinwandt, On the security of two public key cryptosystems using non-abelian groups. Des. Codes Cryptogr. 32 (2004), 207-216. Zbl 1050.94008 MR 2072327

[GW06] D. Gonçalves and P. Wong, Twisted conjugacy classes in wreath products. Internat. J. Algebra Comput. 16 (2006), 875-886. Zbl 1150.20014 MR 2274719

[Gri84] R. I. Grigorchuk, Degrees of growth of finitely generated groups, and the theory of invariant means. Izv. Akad. Nauk SSSR Ser. Mat. 48 (1984), 939-985; English transl. Math. USSR-Izv. 25 (1985), 259-300. Zbl 0583.20023 MR 0764305

[Gri85] R. I. Grigorchuk, A connection between algorithmic problems and entropy characteristics of groups. Dokl. Akad. Nauk SSSR 284 (1985), 24-29; English transl. Soviet Math. Dokl. 32 (1985), 355-360. Zbl 0596.20022 MR 806660

[Gro81] M. Gromov, Groups of polynomial growth and expanding maps. Inst. Hautes Études Sci. Publ. Math. 53 (1981), 53-73. Zbl 0474.20018 MR 623534

[Gub86] V. S. Guba, Equivalence of infinite systems of equations in free groups and semigroups to finite subsystems. Mat. Zametki 40 (1986), 321-324; English transl. Math. Notes 40 (1986), 688-690. Zbl 0611.20020 MR 869922

[Guy07] L. Guyot, Estimations de dimensions de Minkowski dans l'espace des groupes marqués. Ann. Fac. Sci. Toulouse Math. (6) 16 (2007), 107-124. Zbl 1191.20040 MR 2325594

[GS08] L. Guyot and Y. Stalder, Limits of Baumslag-Solitar groups. Groups Geom. Dyn. 2 (2008), 353-381. Zbl 1230.20028 MR 2415304

[Har85] P. de la Harpe, Reduced $C^{*}$-algebras of discrete groups which are simple with a unique trace. In Operator algebras and their connections with topology and ergodic theory (Bussteni, 1983), Lecture Notes in Math. 1132, Springer-Verlag, Berlin 1985, 230-253. Zbl 0575.46049 MR 799571

[Har07] P. de la Harpe, On simplicity of reduced $C^{*}$-algebras of groups. Bull. London Math. Soc. 39 (2007), 1-26. Zbl 1123.22004 MR 2303514

[Har08] P. de la Harpe, Spaces of closed subgroups of locally compact groups. Preprint 2008. arXiv:0807.2030

[HB00] P. de la Harpe and M. Busher, Free products with amalgamation, and HNNextensions of uniformly exponential growth. Mat. Zametki 67 (2000), 811-815; English transl. Math. Notes 67 (2000), 686-689. Zbl 0998.20025 MR 1820635

[HP11] P. de la Harpe and J-P. Préaux, $C^{*}$-simple groups: amalgamated free products, HNN-extensions and fundamental groups of 3-manifolds. J. Topol. Anal. 3(2011), 451-489. Zbl 06017820 MR 2887672 
[HS65] J. Hartmanis and R. E. Stearns, On the computational complexity of algorithms. Trans. Amer. Math. Soc. 117 (1965), 285-306. Zbl 0131.15404 MR 0170805

[Hol00] D. F. Holt, Word-hyperbolic groups have real-time word problem. Internat. $J$. Algebra Comput. 10 (2000), 221-227. Zbl 1083.20507 MR 1758286

[HRR+05] D. F. Holt, S. Rees, C. E. Röver, and R. M. Thomas, Groups with context-free co-word problem. J. London Math. Soc. (2) 71 (2005), 643-657. Zbl 1104.20033 MR 2132375

[HR03] D. F. Holt and C. E. Röver, On real-time word problems. J. London Math. Soc. (2) 67 (2003), 289-301. Zbl 1048.03034 MR 1956136

[HU79] J. E. Hopcroft and J. D. Ullman, Introduction to automata theory, languages, and computation. Addison-Wesley Publishing Co., Reading, Mass., 1979. Zbl 0426.68001 MR 645539

[Iva07] N. A. Ivanov, On the structure of some reduced amalgamated free product $C^{*}$ algebras. Internat. J. Math. 22 (2011), 281-306. Zbl 1239.46040 MR 2782689

[JS79] W. H. Jaco and P. B. Shalen, Seifert fibered spaces in 3-manifolds. Mem. Amer. Math. Soc. 21 (1979), no. 220. Zbl 0415.57005 MR 539411

[KS71] A. Karrass and D. Solitar, Subgroups of HNN groups and groups with one defining relation. Canad. J. Math. 23 (1971), 627-643. Zbl 0232.20051 MR 0301102

[LZ77] R. J. Lipton and Y. Zalcstein, Word problems solvable in logspace. J. Assoc. Comput. Mach. 24 (1977), 522-526. Zbl 0359.68049 MR 0445901

[LS77] R. C. Lyndon and P. E. Schupp, Combinatorial group theory. Ergeb. Math. Grenzgeb. 89, Springer-Verlag, Berlin 1977. Zbl 0368.20023 MR 0577064

[MO85] K. Madlener and F. Otto, Pseudo-natural algorithms for the word problem for finitely presented monoids and groups. J. Symbolic Comput. 1 (1985), 383-418. Zbl 0591.20038 MR 849044

[Mah46] K. Mahler, On lattice points in $n$-dimensional star bodies. I. Existence theorems. Proc. Roy. Soc. London. Ser. A. 187 (1946), 151-187. Zbl 0060.11710 MR 0017753

[Mil71] C. F. Miller, III, On group-theoretic decision problems and their classification. Ann. of Math. Stud. 68, Princeton University Press, Princeton, N.J., 1971. Zbl 0277.20054 MR 0310044

[Mol69] D. I. Moldavanskiı̆, A certain theorem of Magnus. Ivanov. Gos. Ped. Inst. Učen. Zap. 44 (1969), 26-28. MR 0316577

[MS83] D. E. Muller and P. E. Schupp, Groups, the theory of ends, and context-free languages. J. Comput. System Sci. 26 (1983), 295-310. Zbl 0537.20011 MR 710250

[MR00] A. Myasnikov and V. Remeslennikov, Algebraic geometry over groups. II. Logical foundations. J. Algebra 234 (2000), 225-276. Zbl 0970.20017 MR 1799485

[Nek07] V. Nekrashevych, A minimal Cantor set in the space of 3-generated groups. Geom. Dedicata 124 (2007), 153-190. Zbl 05180055 MR 2318543

[OH07] A. Ould Houcine, Limit groups of equationally Noetherian groups. In Geometric group theory, Trends Math., Birkhäuser, Basel 2007, 103-119. Zbl 1162.20029 MR 2395792 
[Pow75] R. T. Powers, Simplicity of the $C^{*}$-algebra associated with the free group on two generators. Duke Math. J. 42 (1975), 151-156. Zbl 0342.46046 MR 0374334

[Rog70] C. A. Rogers, Hausdorff measures. Cambridge University Press, London 1970. Zbl 0204.37601 MR 0281862

[Rom] V. Roman'kov, Twisted conjugacy classes in nilpotent groups. J. Pure Appl. Algebra 215 (2011), 664-671. Zbl 1222.20025 MR 2738380

[Seg83] D. Segal, Polycyclic groups. Cambridge Tracts in Math. 82, Cambridge University Press, Cambridge 1983. Zbl 0516.20001 MR 713786

[Ser77] J.-P. Serre, Arbres, amalgames, SL 2. Astérisque 46 (1977). Zbl 0369.20013 MR 0476875

[Sha00] Y. Shalom, Rigidity of commensurators and irreducible lattices. Invent. Math. 141 (2000), 1-54. Zbl 0978.22010 MR 1767270

[Sta05] Y. Stalder, Espace des groupes marqués et groupes de Baumslag-Solitar. Ph.D. thesis, Université de Neuchâtel, 2005. $\mathrm{http}: / / \mathrm{doc} \cdot$ rero.ch/search.py?recid=5501\&ln=en

[Sta06a] Y. Stalder, Convergence of Baumslag-Solitar groups. Bull. Belg. Math. Soc. Simon Stevin 13 (2006), 221-233. Zbl 05232547 MR 2259902

[Sta06b] Y. Stalder, Moyennabilité intérieure et extensions HNN. Ann. Inst. Fourier (Grenoble) 56 (2006), 309-323. Zbl 1143.20013 MR 2226017

[Ste96] A. M. Stepin, Approximation of groups and group actions, the Cayley topology. In Ergodic theory of $\mathbb{Z}^{d}$ actions (Warwick, 1993-1994), London Math. Soc. Lecture Note Ser. 228, Cambridge University Press, Cambridge 1996, 475-484. Zbl 0857.54034 MR 1411234

[Waa81] S. Waack, Tape complexity of word problems. In Fundamentals of computation theory (Szeged, 1981), Lecture Notes in Comput. Sci. 117, Springer-Verlag, Berlin 1981, 467-471. Zbl 0498.03038 MR 653014

[Weh73] B. A. F. Wehrfritz, Two examples of soluble groups that are not conjugacy separable. J. London Math. Soc. (2) 7 (1973), 312-316. Zbl 0272.20048 MR 0338176

[Wei00] K. Weihrauch, Computable analysis. Texts Theoret. Comput. Sci. EATCS Ser., Springer-Verlag, Berlin 2000. Zbl 0956.68056 MR 1795407

[Why01] K. Whyte, The large scale geometry of the higher Baumslag-Solitar groups. Geom. Funct. Anal. 11 (2001), 1327-1343. Zbl 1004.20024 MR 1878322

[ZL70] A. K. Zvonkin and L. A. Levin, The complexity of finite objects and the development of the concepts of information and randomness by means of the theory of algorithms. Uspehi Mat. Nauk 25 (1970), no. 6, 85-127; English transl. Russian Math. Surveys 25 (1970), no. 6, 83-124. Zbl 0222.02027 MR 0307889

Received February 2, 2010; revised November 24, 2010

L. Guyot, Mathematisches Institut, Georg-August Universität, Bunsenstrasse 3-5,

37073 Göttingen, Germany

E-mail: Luc.Guyot@epfl.ch 
Y. Stalder, Laboratoire de Mathématiques, Université Blaise Pascal, Campus Universitaire des Cézeaux, 63177 Aubière Cedex, France

E-mail: yves.stalder@math.univ-bpclermont.fr 